\title{
Profiling the endothelial translatome in vivo using 'AngioTag' zebrafish
}

Mayumi Miller ${ }^{1}$, Derek E. Gildea ${ }^{2}$, Kathryn Monzo ${ }^{1}$, Lisa Price ${ }^{1}$, Van N. Pham ${ }^{1}$, Natalie Aloi ${ }^{1}$, Amber N. Stratman ${ }^{1,3}$, Andrew Davis ${ }^{1}$, Andreas D. Baxevanis ${ }^{2}$, and Brant M. Weinstein $^{1}$

1. Division of Developmental Biology, Eunice Kennedy Shriver National Institute of Child Health and Human Development, National Institutes of Health, Bethesda, MD 20892

2. Computational and Statistical Genomics Branch, Division of Intramural Research, National Human Genome Research Institute, National Institutes of Health, Bethesda, MD 20892

3. Current Address: Department of Cell Biology and Physiology, Washington University School of Medicine, St. Louis, MO

Correspondence: Brant M. Weinstein, Division of Developmental Biology, Eunice Kennedy Shriver National Institute of Child Health and Human Development, National Institutes of Health, Bethesda, MD, 20892

E-mail: flyingfish2@nih.gov

Key Words: AngioTag, RiboTag, Translating Ribosome Affinity Purification, Translatome, RNAseq, Next Generation Sequencing, zebrafish 
bioRxiv preprint doi: https://doi.org/10.1101/815696; this version posted October 27, 2019. The copyright holder for this preprint (which was not certified by peer review) is the author/funder. All rights reserved. No reuse allowed without permission.

AngioTag expression profiling

Page 2

Miller et al.

\section{HIGHLIGHTS}

- An 'AngioTag' transgenic line permits in vivo endothelial expression profiling

- The AngioTag line is used for Translating Ribosome Affinity Purification - RNAseq

- A 'UAS:RiboTag' line enables profiling of any zebrafish cell and tissue type 


\section{SUMMARY}

Vascular endothelial cells in vivo are exquisitely regulated by their local environment, which is absent or disrupted when using methods such as FACS or in vitro cell culture to study native signaling pathways. Here, we profile the gene expression patterns of undisturbed endothelial cells in living animals using a novel 'AngioTag' zebrafish transgenic line that permits isolation of actively translating mRNAs from endothelial cells in their native environment. This transgenic line uses the endothelial cell-specific $k d r l$ promoter to drive expression of an epitope tagged Rpl10a 60S ribosomal subunit protein, allowing for Translating Ribosome Affinity Purification (TRAP) of actively translating endothelial cell mRNAs. By performing TRAP-RNAseq on AngioTag animals, we demonstrate strong enrichment of endothelial specific genes and uncover novel endothelially expressed genes. Additionally, we generated a 'UAS:RiboTag' transgenic line to allow for the study of a wider array of zebrafish cell and tissue types using TRAPRNAseq methods. This new tool offers an unparalleled resource to study cause and effect relationships in the context of gene loss or gain of function in vivo. 


\section{INTRODUCTION}

The vascular system is a complex network of arteries, veins, and capillaries working in concert to allow oxygenated blood to flow throughout the body and transport hormones and small molecules to target tissues. This network is comprised of lumenized endothelial cell tubes surrounded by smooth muscle cells (in larger vessels, especially arteries) or pericytes juxtaposed to the basement membrane. Endothelial cells in vascular tubes have extensive cell-cell junctional contacts with one another and with the surrounding vascular smooth muscle cells. In addition, the luminal surface of the endothelium is constantly exposed to flow dynamics, circulating cells, and circulating factors from the blood, while the abluminal surface interacts extensively with extracellular matrix and with numerous non-vascular cell types and tissues. Understanding how this complex multitude of external cues and signals impinging on endothelial cells work together to regulate blood vessel growth and function is of enormous interest given the important roles vessels play in a variety of pathologies including cancer, ischemia, and congenital vascular disorders.

The earliest precursors of the vascular system are the mesodermally derived hemangioblast progenitors which differentiate into endothelial and hematopoietic lineages (Huber et al., 2004; Vogeli et al., 2006). Endothelial receptor tyrosine kinases (RTKs) such as VEGFR1/2 and Tie1/2 play key roles in both the specification of endothelial cells and the development of endothelial cells into a functional vascular network. Loss of VEGFR2/Flk1 in mice results in the absence of blood vessels and a severe reduction in hematopoetic cells (Shalaby et al., 1995), while loss of VEGFR1/Flt1 results in disorganized vasculature and early embryonic mortality (Fong et al., 1995). The VEGF ligands for VEGFR receptors are expressed in many different surrounding cells and tissues; they are regulated by a variety of genetically programmed and environmental cues such as oxygen and nutrient availability. Loss of just one allele of the VEGF ligand results in embryonic lethality, with vascular and hematopoetic defects (Carmeliet et al., 1996; Dumont et al., 1995; Ferrara et al., 1996). The early vascular network does develop in Tie1 and Tie 2 null mutants. In Tie2 mutants, vessels do not remodel and have low smooth muscle coverage while, in Tie1 mutants, vasculature develops but the integrity of vessels is compromised, resulting in a leaky vasculature (Sato et al., 1995). Angiopoietin ligands for Tie1 and Tie2 are expressed in the mesenchyme surrounding the developing vasculature (Pham et al., 2001).

The complementary expression of VEGFR and Tie receptors (and their VEGF and angiopoietin ligands) in endothelial cells and their surrounding environment provides a few examples of the exquisite regulation of endothelial cells in vivo. In addition to direct circulating and extravascular signals sensed by endothelial cells, cell-cell contacts, cellmatrix contacts, oxygen tension, nutrient availability, and other cues in the local environment all influence endothelial cell function in vivo. Existing methods for analysis of endothelial gene expression and function most frequently rely on post-mortem isolation and/or in vitro culture of endothelial cells, disrupting or preventing normal in vivo interactions experienced by endothelial cells. Endothelial cells have been studied in their endogenous setting using a number of different vertebrate model organisms, including mice, avians, and zebrafish. Zebrafish is a particularly useful model organism for studying vascular development, as its optical transparency, external development, and the availability of transgenic reporter lines expressing fluorescent proteins in the endothelium permits high-resolution visualization of the complex and widely dispersed vascular 
network at all stages of early development (Gore et al., 2012; Jung et al., 2016). These features, along with the ability to house large numbers of adult animals and obtain large numbers of progeny, facilitate genetic and experimental analysis of vascular development in the fish. The advantages of the zebrafish model have led to important discoveries including mechanisms of arterial-venous differentiation (Lawson et al., 2001; Leslie et al., 2007; Swift et al., 2014; Swift and Weinstein, 2009), lumen formation (Jin et al., 2005; Kamei et al., 2006; Yu et al., 2015), and guidance and patterning of vessel networks during development (Childs et al., 2002; Torres-Vazquez et al., 2004).

Although zebrafish and other animal models have been invaluable for probing endothelial function in vivo, existing methods for quantitative global analysis of endothelial gene expression, such as microarrays or RNAseq, still require isolation of endothelial cells from animals prior to profiling. Fluorescence Activated Cell Sorting (FACS) has been used previously to enrich zebrafish endothelial cells for gene expression profiling by microarrays (Cannon et al., 2013; Covassin et al., 2006; Sumanas et al., 2005). In these studies, transgenic zebrafish embryos with fluorescently "tagged" endothelium were enzymatically and mechanically dissociated into single cells suspensions that were then subjected to lengthy FACS sorting before RNA preparation from the enriched endothelial cell population. It is likely that these manipulations, and the disruption of normal interactions over an extended time period, introduce signaling pathway changes, including activation of cell stress and apoptotic pathways, that obscure the native transcriptional state of endothelial cells.

Translating Ribosome Affinity Purification (TRAP; (Sanz et al., 2009) involves collection of actively translating mRNA species via immunoprecipitation of attached ribosomes that are translating these mRNAs. Studies in mice have demonstrated that transgene-driven expression of an epitope-tagged ribosomal protein subunit (also known as a 'RiboTag') within specific cell types or tissues can be used to affinity purify and profile actively translating mRNAs in vivo by performing TRAP followed by RNAseq (TRAP-RNAseq) or microarray experiments (Allison et al., 2015; Hupe et al., 2014; Sanz et al., 2009). We have now developed zebrafish transgenic lines that permit profiling of global gene expression in either vascular endothelial cells ('AngioTag') or in any cell or tissue type for which a Gal4 driver line is available ('UAS:RiboTag'). These lines utilize the vascular endothelial kdrl or UAS promoters, respectively, to drive co-expression of HA-tagged rpl10a, a 60S ribosomal subunit protein, and EGFP (as a visible transgene marker), permitting immunoprecipitation of tissue-specific ribosomes and collection of the mRNAs they are translating. Since isolation of vascular mRNA from these transgenic lines is extremely rapid (embryos are quickly Dounce-homogenized in a lysis buffer containing cycloheximide to halt further translation), the mRNAs collected represent the bona fide expression profiles of undisrupted cells in situ. These transgenic lines provide powerful new tools for profiling cell- or tissue-specific gene expression in the zebrafish. 


\section{RESULTS}

\section{Translating ribosome affinity purification (TRAP) in zebrafish embryos}

To determine whether Translating Ribosome Affinity Purification (TRAP) could be used to isolate polysome-bound mRNAs from developing zebrafish, we designed an egfp-2arpl10a3xHA RiboTag cassette. This cassette includes 60S ribosomal protein 110a (rpl10a) fused to triplicate hemagglutinin (HA) epitope sequences (rpl10a3xHA). To visualize transgene expression, the cassette also includes EGFP linked to rpl10a3xHA via a viral 2A cleavage site (Provost et al., 2007), permitting bicistronic expression of separate EGFP and rpl10a3xHA polypeptides (Figure 1A). Assembly of Rpl10a3xHA protein into functional ribosomes 'marks' translating mRNAs in cells expressing this transgene (Figure 1B). To test whether the transgene could be employed to isolate tagged polysomes using TRAP, we injected RiboTag mRNA into single-cell embryos, raised the embryos to $24 \mathrm{hpf}$, homogenized them, and affinity-purified HA-tagged polysomes using $\alpha H A$ antibody followed by direct immunoprecipitation with Dynabeads ${ }^{\circledR}$ (Figure 1C). Western blotting of starting lysates, post-immunoprecipitation supernatants, and eluates from $\alpha \mathrm{HA}$ pulldowns (Figure 1D) showed that Rpl10a3xHA protein was readily detected in RiboTaginjected (lane 2) but not in control uninjected (lane 1) embryos, and that Rpl10a3xHA protein was efficiently pulled down using the $\alpha \mathrm{HA}$ antibody (lane 6) and depleted from the supernatant (lane 5).

To ensure that Rpl10a3xHA protein is properly incorporated into the $60 \mathrm{~S}$ ribosomal subunit, the $80 \mathrm{~S}$ ribosome, and polysomes, we prepared lysates from RiboTag mRNAinjected and control uninjected embryos and performed velocity sedimentation in sucrose density gradients, followed by Western blotting of collected fractions (Figure 1E,F). We probed the blots using an antibody recognizing endogenous Rpl11, a component of the 60S subunit, and found that Rpl11 sediments similarly within the 60S, 80S, and polysome fractions in either uninjected controls or RiboTag-injected animals (Figure 1E). When we probed the blots using an $\alpha \mathrm{HA}$ antibody, we observed similar sedimentation patterns for Rp110a3xHA protein in the RiboTag-injected animals, leading us to conclude that the epitope tag does not interfere with assembly of the 60S subunit, 80S ribosome, or polysomes (Figure 1E). As expected, disassembly of polysomes using EDTA treatment causes both Rpl11 and Rpl10a3xHA to elute only in the 60S fraction; they are not seen in the $80 \mathrm{~S}$ or polysome fractions (Figure 1F). These results show that Rpl10a3xHA protein assembles into ribosomes in vivo and can be used for isolation of tagged polysomes from zebrafish.

\section{Isolating endothelial-specific RNA using AngioTag zebrafish}

We next set out to create transgenic zebrafish expressing the RiboTag construct specifically within endothelial cells. We utilized the previously published endothelial specific $7 \mathrm{~kb} \mathrm{kdrl}$ promoter (Choi et al., 2007) to design a IsceI(kdrl:egfp-2a-rpl10a3xHA) AngioTag transgene expressing the RiboTag cassette in an endothelial cell type-specific manner (Figure 1G). We obtained a strongly expressing $\mathrm{Tg}(\mathrm{kdrl} \text { :egfp-2a-rpl10a3xHA})^{y 530}$ germline transgenic line that shows a vascular-restricted EGFP expression closely matching the expression pattern of a previousy generated $\mathrm{Tg}(\mathrm{kdrl}: \mathrm{egfp})^{l a 116}$ transgenic line driving EGFP alone (Figure 1H,I). To test whether this AngioTag transgenic line could be used for TRAP enrichment of endothelial mRNAs, we prepared homogenates from 24 
hpf AngioTag or control nontransgenic animals and subjected the lysates to the TRAP procedure (Figure 1J). Western blotting of starting lysates (lanes 1 and 2), TRAP supernatants (lanes 3 and 5), and TRAP eluates (purified tagged polysomes, lanes 4 and 6) from non-transgenic (lanes 1,3,4) or AngioTag transgenic (lanes 2,5,6) animals (Figure 1K) showed that Rpl10a3xHA protein was readily detected in AngioTag (lane 2) but not in control (lane 1) non-transgenic total lysates, and that Rpl10a3xHA was pulled down from AngioTag lysates using $\alpha \mathrm{HA}$ antibody (lane 6). To assess whether the AngioTag TRAP procedure resulted in selective enrichment of endothelial-specific mRNAs, we performed quantitative RT-PCR for endothelial and non-endothelial genes using RNA collected from either $24 \mathrm{hpf}$ AngioTag transgenic animals or $24 \mathrm{hpf}$ RiboTag mRNA injected animals. RNA isolated from AngioTag animals was highly enriched for endothelial-specific genes $(c d h 5, k d r l)$ and depleted of genes expressed specifically in nonvascular tissues (desmin, ngnl) compared to the RiboTag mRNA injected control RNA samples (Figure 1L).

\section{Expression profiling using TRAP-RNAseq in zebrafish}

Having shown that our AngioTag transgenic line could be used to selectively enrich for endothelial gene expression, we wanted to compare AngioTag TRAP-RNAseq endothelial expression profiling to FACS endothelial expression profiling using previously published methods in zebrafish (Cannon et al., 2013; Covassin et al., 2006; Sumanas et al., 2005) (Figure 2A,B). As noted above, FACS protocols require the dissociation of an embryo into a single cell suspension, disrupting cell-cell, cell-matrix, and other external interactions for a prolonged period of time prior to cell lysis and RNA collection (Figure 2A). In contrast, the TRAP procedure involves immediate homogenization, cell lysis, and stabilization of translating RNAs (Figure 2B), providing an instant 'snapshot' of translated gene expression at the time of embryo homogenization, one that is potentially more representative of an 'in vivo' profile.

We performed RNAseq in triplicate on a total of six different sample types (Figure 2C). RNA was prepared from TRAP-purified polysomes from $24 \mathrm{hpf}$ RiboTag mRNA injected animals (Sample 2, 'whole TRAP translatome'), from FACS-sorted endothelial cells from $24 \mathrm{hpf}$ AngioTag transgenic animals (Sample 4, 'endothelial FACS transcriptome'), and from TRAP-purified polysomes from $24 \mathrm{hpf}$ AngioTag transgenic animals (Sample 6, 'endothelial TRAP translatome'). For each of these samples, we also collected control RNA from either the starting lysates used for TRAP (Samples 1 and 5) or from unsorted cells (Sample 3). Three experimental replicates were performed for each of these sample types from separately collected samples, for a total of 18 samples used for RNAseq analysis. A minimum of 45 million reads were obtained for each sample, with an average of $84 \%$ of reads mapping uniquely (Supp. Table 1). All sequence data was deposited with GEO (accession \# GSE121973). Principal component analysis (Figure 2D) showed that the RiboTag-TRAP $(1,2)$ and AngioTag-TRAP $(5,6)$ starting lysates and TRAP samples clustered fairly closely together, while the FACS unsorted (3) and sorted (4) cell samples showed considerable divergence both from the other samples and from one another.

Comparing the whole-embryo lysate transcriptome (Sample 1) and matched whole-embryo TRAP translatome (Sample 2) from animals ubiquitously expressing RiboTag (Figure 3A) showed that 1459 genes were significantly differentially represented in the two RNAseq data sets (fold $\geq 1.5$ or $\leq 1.5$, Benjamini-Hochberg $(\mathrm{BH})$-adjusted $\mathrm{p}<0.05$ ), with 829 genes 
showing increased representation and 630 genes showing reduced representation in the whole-embryo TRAP translatome (Sample 2) vs. transcriptome (Sample 1) data sets. Nine of the ten PANTHER overrepresentation test (Mi et al., 2013) GO terms with the largest fold increase in the whole animal translatome (Sample 2) vs. the transcriptome (Sample 1) were related to splicing and/or RNA metabolism, suggesting mRNAs for these genes may be more highly translated (Figure 3B). To determine whether there are any sequence features associated with increased or decreased translation, we examined relative codon usage associated with genes over- or under-represented in sample 2 vs. sample 1, identifying strong preferences for and against particular codons in more- versus less-highly translated genes (Figure 3C). Interestingly, our findings closely align with previously published data examining codon usage bias in highly transcribed genes in the zebrafish genome (Horstick et al., 2015). We also examined the 3' UTR sequence length and found that more highly translated genes have, on average, shorter 3' UTRs (Figure 3D), in agreement with prior work suggesting mRNAs with long 3' UTRs have increased numbers of miRNA binding sites leading to lower levels of translation (Schwanhausser et al., 2011; Vogel et al., 2010).

\section{AngioTag profiling endothelial genes}

Having shown that TRAP-RNAseq can be used effectively to profile translating mRNAs in vivo in the zebrafish, we next sought to examine whether our AngioTag transgenic line could be used for specific profiling of endothelium in vivo. As noted above, FACS has been used previously to isolate endothelial cells from developing zebrafish for gene expression profiling (Cannon et al., 2013; Covassin et al., 2006; Sumanas et al., 2005) so, for purposes of comparison, we began by carrying out RNAseq analyses on mRNA from endothelial cells isolated from $24 \mathrm{hpf}$ AngioTag animals by FACS (Figure 4). RNAseq experiments were carried out on mRNA isolated from total dissociated cells from $24 \mathrm{hpf}$ AngioTag embryos (Sample 3) and on mRNA from EGFP-positive endothelial cells FACS-sorted from the same dissociated cell population (Sample 4; Figure 4A). Comparative analysis of genes represented in the two RNAseq data sets showed that 3359 genes were significantly enriched in the FACS-sorted endothelial cells (fold $\geq 1.5$, BHadjusted $\mathrm{p} \leq 0.05$ ), while 3173 genes showed significant depletion in the FACS-sorted endothelial cell population compared to the total dissociated cell population (fold $\leq 1.5$, BH-adjusted $\mathrm{p} \leq 0.05$; Figure 4B). Six out of the top 20 PANTHER overrepresentation test GO terms showing the highest fold enrichment in FACS-sorted endothelial cells were endothelial/vascular-related terms, while th e other 14 most highly enriched GO terms were non-endothelial (Figure 4C).

Next, we examined the results of profiling the endothelium in the AngioTag line using TRAP-RNAseq (Figure 5). We compared RNAseq data sets of whole animal translatome (Sample 2) and endothelial translatome (Sample 6) after normalization of each of these samples to their whole-animal starting lysate transcriptome (samples 1 and 5, respectively; Figure 5A). 2135 genes were enriched in the endothelial translatome (fold $\geq 1.5$, BHadjusted $\mathrm{p} \leq 0.05$ ), while 1868 genes show some depletion when compared to the whole animal translatome (fold $\leq 1.5$, BH-adjusted $\mathrm{p} \leq 0.05$; Figure 5B). Of the top $20 \mathrm{GO}$ terms enriched in the endothelial TRAP-RNAseq data set, 14 were endothelial/vascular-related terms (Figure 5C), a substantially higher number than found in the FACS-sorted endothelial cell data (Figure 4C). Although six of these $14 \mathrm{GO}$ terms were also present in 
the top 20 terms for the FACS-sorted endothelial cells, they showed greater foldenrichment with lower p-values in the TRAP-RNAseq data set (Supp. Table 2). Known endothelial-specific genes are highly enriched in the AngioTag TRAP-RNAseq data set, while genes that are not expressed in endothelium are depleted (Figure 5D).

The vast majority of genes highly enriched by AngioTag TRAP-RNAseq are annotated, and many of these genes are already known to be expressed in the endothelium. However, our enriched gene set also includes unannotated genes lacking a previously reported link to endothelium, including four genes found amongst the 50 most highly enriched genes in our data set (Figure 5E), each of which shows at least 20-fold endothelial enrichment (Figure 5F). Whole mount in situ hybridization confirms that all four of these genes have highly endothelial-specific expression patterns (Figure 5G-N), with expression of one of the genes largely restricted to the endothelium of the caudal vascular plexus (Figure $\mathbf{5 H}, \mathbf{L}$ ). In addition to unannotated genes, some of the annotated genes in our highly enriched gene set also lack previously reported expression in endothelium. Examination of four of these genes (Figure 50,P) by in situ hybridization also reveals an endothelial-specific expression pattern (Figure 5Q-T). To explore the functional significance of a few of the un-annotated genes, we use CRISPR/Cas9 technology to generate targeted mutations in Gene B (ENSDARG00000076721) and in Gene C (ENSDARG00000098293). We isolated a 5 base pair deletion mutant in gene B $\left(76721^{\mathrm{y} 587}\right)$ generating a polypeptide truncated after 77 amino acids (Figure 6A), and a 20 base pair deletion mutant in gene $\mathrm{C}$ (98293 ${ }^{\text {y58 }}$ ) coding for a polypeptide truncated after 25 amino acids (Figure 6F). Homozygous $76721^{\mathrm{y} 587}$ mutants display a mild enlargement of the caudal vascular plexus (Figure 6B-E), while homozygous $98293^{\mathrm{y} 588}$ mutants show reduced growth of mid- and hindbrain cranial central arteries (Figure 6E-G). These results suggest that at least two of the four novel, un-annotated genes from this study have roles in early vascular development.

Together, our results suggest that TRAP-RNAseq using our AngioTag transgenic line provides a highly effective method for in vivo profiling of endothelial gene expression.

\section{A RiboTag reporter line for profiling cells and tissues of interest}

The success of endothelial profiling using AngioTag TRAP-RNAseq led us to ask whether we could generalize this method to profile other, non-endothelial tissues. In order to do this, we generated a $\operatorname{Tg}$ (uas:egfp-2a-rpl10a2xHA) ${ }^{y 531}$ UAS:RiboTag transgenic line to drive expression of the RiboTag cassette in any tissue or cell type of interest for which a Gal4 driver line is available (Figure 7A,E). The UAS:RiboTag line showed strong, tissuespecific expression after being crossed to a variety of different Gal4 driver lines, including a muscle-specific $\operatorname{Tg}\left(x a 210:\right.$ gal4$^{y 241}$ line (Figure 7B; (Fero et al., 2014), an endothelialspecific Tg(flila:gal4ff) ${ }^{u b s} 4$ line (Figure 7C; (Zygmunt et al., 2011), and a neural-specific $\mathrm{Tg}(\mathrm{huC}$ :gal4) line (Figure 7D; (Kimura et al., 2008). TRAP isolation of mRNA from embryos obtained from each of these crosses revealed strong enrichment of tissue-specific genes in samples prepared from the appropriate lines (Figure 7E). The enrichment of the endothelial-specific $k d r l$ gene in TRAP-purified mRNA from Tg(uas:egfp-2arpl10a2xHA) ${ }^{y 531}$; $\operatorname{Tg}\left(\right.$ flila:gal4ff) ${ }^{u b s} 4$ double-transgenic animals was comparable to that found in TRAP-purified mRNA from our Tg(kdrl:egfp-2a-rpl10a3xHA $)^{y 530}$ AngioTag line, suggesting that the UAS:RiboTag is similarly effective as a tool for tissue-specific gene expression profiling. 


\section{DISCUSSION}

In this study, we introduce a novel AngioTag transgenic line for profiling global gene expression in zebrafish vascular endothelial cells in their undisturbed endogenous environment. We have performed TRAP-RNAseq experiments using AngioTag fish to profile the endothelial translatome, comparing the results to RNAseq data obtained from FACS-isolated endothelial cells and demonstrating that the TRAP-RNAseq approach results in superior endothelial enrichment. Using our transgenic tool, we have identified several novel unannotated vascular genes, and we have also documented vascular enrichment of a number of annotated genes that were not previously known be expressed in the endothelium. Finally, to make TRAP-RNAseq methodologies accessible to all researchers, not just those studying the vascular endothelium, we have generated a UASRiboTag line that can be used to profile any cell and tissue type for which a Gal4 driver is available.

Classically, translatomes have been prepared for profiling using sucrose density gradients, collecting RNA from the polysome fraction (Wiegers and Hilz, 1972). Profiling the translatome of a particular tissue type using this method requires either dissection or FACS sorting. More recently, the TRAP method has been used to profile the translatomes of specific tissues or cell types by using mice with a floxed conditional 'tagged' allele of the Rp122 ribosomal protein expressed after crossing the mice to cell- or tissue-specific cre driver lines (Sanz et al., 2009). RiboTag mice have been used to assay gene expression in hypothalamic neurons through TaqMan assays (Soden et al., 2016), sertoli and leydig cells of the testes through microarrays (Sanz et al., 2013), and proneural gliomas through ribosomal footprinting on translatome RNA (Gonzalez et al., 2014). Other groups have utilized the TRAP method to perform RNAseq on specific cell populations in the mouse brain or kidney, with all but one group amplifying their RNA prior to sequencing (Brichta et al., 2015; Hupe et al., 2014; Mardinly et al., 2016; Shigeoka et al., 2016). Tissue specific RiboTag zebrafish have also been generated driving expression of GFP fused to rpl10a with tissue-specific promoters and then utilizing anti-GFP antibodies to immunoprecipitate tissue specific translatomes. Translatomes prepared in this way have been used to assay gene expression in the heart using microarrays (Fang et al., 2013) and in melanocytes using qPCR (Tryon et al., 2013). A binary zebrafish RiboTag system has also been generated in which transgenic zebrafish carrying Avi-tagged Rpl10a are crossed to tissue specific BirA lines to biotinylate Rp110a in BirA-expressing tissues. This binary biotinylation method was used to perform microarray analysis on zebrafish skeletal muscle mRNAs (Housley et al., 2014). Notably, the two zebrafish studies noted above using microarrays also amplified their collected translatome RNA prior to further analysis. In contrast, our TRAP-RNAseq procedures were performed without any amplification of collected whole embryo or endothelial translatome RNA.

Our whole embryo translatome TRAP-RNAseq results provide a valuable data set for uncovering new insights into sequence features associated with more- or less-highly translated genes. Interestingly, our data on codon usage associated with highly translated genes shows strong parallels with previously published data examining codon usage in the developing zebrafish (Horstick et al., 2015). In that study, the authors examined codon usage in the 469 most highly transcribed genes in the zebrafish genome (as determined from previously published RNAseq data), identifying codons preferentially associated with 
highly transcribed genes. These authors went on to show that by replacing the native codons with codons preferred in "more transcribed" genes they were able to increase protein accumulation, providing experimental support for the idea that zebrafish use "optimal" codons to increase translation of their already highly transcribed genes. Our data reveals that many of the same codons enriched in the highly transcribed gene set of Horstick et al. are also preferentially enriched in the most-highly translated genes in our whole embryo translatome data set (Fig. 3C), providing direct experimental evidence for the idea that highly transcribed genes are also "translationally optimized."

Comparison between our TRAP-RNAseq (Fig. 5) and FACS-RNAseq (Fig. 4) endothelial data sets suggests that TRAP-RNAseq provides more effective enrichment of endothelial genes. Of the top 20 enriched GO terms specific to each data set, analysis on the TRAPRNAseq data identified enrichment of more than twice as many vascular-related biological process GO terms as in the FACS-RNAseq data set. (Supp. Table 2). Interestingly, principal component analysis of our RNAseq data (Fig. 2D) also shows that the three AngioTag TRAP-RNAseq replicates (red spheres) are more highly clustered with one another and with the other non-FACS RNAseq data sets (light blue, dark blue, and yellow spheres) than the RNAseq data from either FACS-sorted endothelial cells (green spheres) or from dissociated but unsorted total embryonic cells (purple spheres), which are much less clustered, either with their own replicates or with the other RNAseq data sets. These results suggest that technical aspects of the FACS process, such as embryonic dissociation and prolonged incubation of separated cells prior to collection of mRNA (samples 3 and 4 in Fig. 2C), introduce significant changes in gene expression compared to samples in which cells are rapidly disrupted prior to either immediate collection of mRNA (samples 1 and 5 in Fig. 2C) or TRAP-RNAseq (samples 2 and 6 in Fig. 2C). Utilizing our TRAP-RNAseq methods, we identified previously uncharacterized endothelial genes. These include both previously un-annotated genes (Fig. 5E-N) and annotated genes that have not hitherto been shown to have endothelial-enriched expression (Fig. 5O-T), some of which we have also shown are required for normal vascular development (Fig. 6). These results further reinforce the idea that the AngioTag TRAP-RNAseq approach can provide unique insights compared to FACS sorting of dissociated endothelial cells.

The new $\operatorname{Tg}$ (uas:egfp-2a-rpl10a2xHA $)^{y 531}$ UAS:RiboTag line that we have generated in the course of this work makes it possible to apply the TRAP-RNAseq methods we used to profile the endothelium to any cell or tissue type in the zebrafish for which a Gal4 driver line is available (Fig. 7). A very large number of transgenic Gal4 driver lines have been generated in the zebrafish with expression in a wide assortment of different cells and tissues, including many lines derived from enhancer trap screens (for example, (Davison et al., 2007; Kawakami et al., 2010; Marquart et al., 2015; Scott et al., 2007). The availability of these lines should allow the wide application of our TRAP-RNAseq methods.

Together, the new tools and methods we have developed for transgene-assisted TRAPRNAseq provide a valuable resource for cell- and tissue-specific in situ expression profiling in the zebrafish. Combined with the many other genetic and experimental tools available for studying development in the zebrafish, we expect these new tools and methods to greatly enhance our capability to analyze signalling pathways in living animals. 


\section{MATERIALS AND METHODS}

\section{Zebrafish}

Zebrafish were maintained and zebrafish experiments were performed according to standard protocols (Westerfield, 2000) and in conformity with the Guide for the Care and Use of Laboratory Animals of the National Institutes of Health, in an Association for Assessment and Accreditation of Laboratory Animal Care (AAALAC) accredited facility.

\section{Sucrose Gradient}

Sucrose density gradients were prepared for sedimentation analysis of polysome profiles.

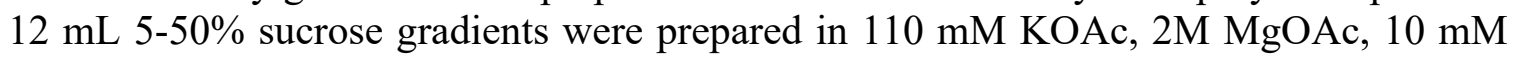
HEPES pH 7.6 with BioComp Gradient Master (BioComp), and allowed to rest overnight at $4^{\circ} \mathrm{C}$. Dechorionated and deyolked embryos were dounce homogenized in $1.5 \mu \mathrm{l}$ polysome fractionation buffer per embryo (10mM HEPES pH7.4, 110mM KOAc, $2 \mathrm{mM}$ MgOAc, $100 \mathrm{mM} \mathrm{KCl}, 10 \mathrm{mM} \mathrm{MgCl}_{2}, 0.1 \%$ Nonidet P-40, 2mM DTT, 40U/mL RNasin, $200 \mathrm{ug} / \mathrm{mL}$ cycloheximide, and protease inhibitors; adapted from (Lai et al., 2009)). Homogenates were centrifuged at $1000 \mathrm{xg}$ for $10 \mathrm{~min}$ at $4^{\circ} \mathrm{C}$. Protein concentration was quantified by Bradford Assay (Sigma Aldrich) and equivalent amounts were loaded onto gradients. Samples were centrifuged in a SW41 Beckman rotor at $4000 \mathrm{rpm}$ at $4{ }^{\circ} \mathrm{C}$ for 2 hours. $16 \mathrm{x} 1 \mathrm{~mL}$ fractions were collected with an ISCO piercing apparatus connected to a BioLogic chromatography system at $0.5 \mathrm{~mL} / \mathrm{min}$, pushing $55 \%$ sucrose. Data was collected using LP DataView software (BioRad). For EDTA treatment, samples were treated with $200 \mathrm{mM}$ final concentration EDTA prior to loading onto gradient.

\section{TRAP protocol}

Translating Ribosome Affinity Purification (TRAP) was performed as described previously (Sanz et al., 2009) with modifications. For each TRAP sample approximately 1200-1500 24 hpf zebrafish embryos per were dechorionated, deyolked, and dounce homogenized in homogenization buffer consisting of $50 \mathrm{mM}$ Tris $\mathrm{pH} 7.4,100 \mathrm{mM} \mathrm{KCl}$, $12 \mathrm{mM} \mathrm{MgCl}_{2}, 1 \%$ NP-40, $1 \mathrm{mM}$ DTT (Sigma, Cat. \#646563), 1x Protease inhibitors (Sigma, Cat. \#P8340), 200 units $/ \mathrm{mL}$ RNAsin (Promega, Cat. \#N2115), 100ug/mL Cycloheximide (Sigma Cat. \#7698), 1mg/mL Heparin (Sigma, Cat. \#H3393-10KU). Lysates were cleared for $10 \mathrm{~min}$ at $10,000 \mathrm{xg}, 4^{\circ} \mathrm{C} .1 \mu \mathrm{l}$ of anti-HA antibody (Abcam ab9110 Rabbit polyclonal) was added per 400 ug protein in an $800 \mu$ l lysate and the samples were orbitally rotated at $4^{\circ} \mathrm{C}$ for 5 hours. $60 \mu 1$ of Dynabeads ${ }^{\circledR}$ protein G slurry (Novex/Life Technologies) was added per $1 \mu$ of anti-HA antibody with homogenization. Before using, Dynabeads were washed with 800ul homogenization buffer, rotating for 30 minutes to equilibrate the beads. The wash buffer was then removed from the Dynabeads and the lysate $+\mathrm{Ab}$ solution was added to the equilibrated Dynabeads. This Dynabead + lysate $+\mathrm{Ab}$ mixture was incubated overnight at $4 \operatorname{deg} \mathrm{C}$ on an orbital rotator. Dynabeads were collected using a magnetic stand, and washed $3 \times 5 \mathrm{~min}$ on an orbital shaker with high salt homogenization buffer (50mM Tris pH 7.4, 300mM KCl, $12 \mathrm{mM} \mathrm{MgCl} 2,1 \%$ NP-40, $1 \mathrm{mM}$ DTT, 1x Protease inhibitors, 200 units $/ \mathrm{mL}$ RNAsin, 100ug/mL Cyclohexamide, $1 \mathrm{mg} / \mathrm{mL}$ Heparin). RNA was collected from the Dynabeads and DNAse treated using the Zymo ZR-Duet Kit (Zymo Research D7003). Note that careful attention to the protocol is required to keep lysates cold and free of RNases, and RNA integrity must be carefully monitored. Large numbers of embryos are needed to provide the starting material for the 
TRAP protocol because (i) the endothelial or other cell types expressing the RiboTag represent a small fraction of the total cells in the animal, (ii) only a fraction of the ribosomes in the targeted cell type contain the RiboTag - most ribosomes are still "untagged," and (iii) TRAP-purified RNA samples contain a large amount of co-purifying ribosomal RNAs in addition to the mRNAs, and (iv) we prepared largely unamplified libraries for sequencing.

\section{Quantitative RT-PCR}

RNA was reverse transcribed using a High Capacity cDNA reverse transcription kit (Applied Biosystems ${ }^{\mathrm{TM}}$ ). cDNA was combined with the primers listed below and with LightCycler ${ }^{\circledR} 480$ SYBR Green I (Roche). Reactions were run in a LightCycler ${ }^{\circledR} 480$ Multiwell Plate 384 machine (Roche). Relative fold changes were calculated using ddCT calculations and by calculating standard deviations. The following primer pairs were used:

\begin{tabular}{ll} 
Gene & Forward Primer \\
\hline cdh5 & CAACAGACGCTGATGATTCC \\
kdrl & GAGTTCCAGCACCCTTTATCA \\
desmin & CGGTGGTTATCAGGACACTATC \\
neurogenin 1 & CGCATTGGATGCTTTGAGAAG \\
snap25 & GGCTACTGTCATGCTCCTTATT \\
actin b1 & CGAGCAGGAGATGGGAACC
\end{tabular}

Reverse Primer

GTCTTTGGCTTGAACAGCAA

ATCGTCCTTCTTCACCCTTTC

TCCAGAGCCATCTTCACATTC

CGAAAGTGCCCAGATGTAGT

TGATTGTAAGTGCTCGTCGTATTA

CAACGGAAACGCTCATTGC

\section{RNAseq}

RNA concentration and quality were measured on a Qubit (Thermo Fisher) and on an Agilent 2100 BioAnalyzer + RNA Pico Chip (Agilent), respectively. Because a large proportion of the RNA samples we collected represent ribosomal RNAs co-purified with our polysome mRNAs, $500 \mathrm{ng}$ RNA was polyA selected or rRNA depleted using RiboZero rRNA Removal Kit (Illumina) before library construction. Sequencing libraries were constructed from the purified mRNA using TruSeq Stranded mRNA Library Prep Kits (Illumina). Libraries were sequenced using an Illumina HiSeq 2500 to generate approximately 50 million $2 \times 75$ bp or $2 \times 100$ bp paired end reads. Raw data were demultiplexed and analyzed. RNAseq data was deposited with GEO, accession \# GSE121973.

\section{Assessing mRNA abundance from RNAseq data}

RNA-seq reads were mapped to the zebrafish reference genome (GRCz10) using the STAR alignment software package (v. 2.4.2a). Only those RNA-seq reads mapping to annotated protein-coding Ensembl gene models were used for gene expression profiling. Quantification of the RNA-seq data was done using the RSEM software suite (v. 1.2.22), obtaining raw and normalized RNA-seq read counts (in transcripts per million, TPM). DESeq2 was used to compare mRNA abundance between samples. For comparing mRNA abundance between TRAP-enriched and FACS-sorted samples, raw RNAseq read counts from enriched samples were normalized to the read counts from RNAseq performed on total mRNA input used for the TRAP enrichment and total mRNA from unsorted cells prior to FACS. Using the RNAseq data from the TRAP input and the unsorted cells prior to FACS, normalization factors were calculated by dividing the TPM abundance for a given mRNA in each sample by the geometric mean of the TPM abundances across all samples. The raw read counts from the TRAP-RNAseq and FACS-RNAseq data sets were divided 
by their corresponding normalization factor to adjust for differences in input mRNA levels prior to enrichment. With these adjusted RNAseq read counts, DESeq2 was used to identify differences in mRNA abundance.

\section{Analysis of gene features}

Ensembl gene models were used to calculate codon usage and 3' UTR lengths. The longest transcript/isoform was selected to represent each annotated Ensembl gene. For calculating codon usage, the coding sequences for all transcripts were obtained from the Ensembl (ftp://ftp.ensembl.org) in FASTA format. A custom Perl script was written to retrieve specific transcript sequences and to count codons from these sequences. For calculating 3' UTR lengths, positional information for Ensembl gene annotations were downloaded from UCSC Table Browser in BED format. The genomic start position for the 3' UTR of each transcript was obtained using the nucleotide position immediately following the end of the annotated coding sequence position. The 3' UTR stop position was obtained from the annotated transcription stop site. The 3' UTR length was calculated using these start and stop positions.

\section{Western blot}

Protein samples were collected and boiled in Laemmli buffer + 2-Mercaptoethanol and run on $12 \%$ polyacrylamide gels, transferred onto PVDF membranes, and then blocked in 5\% BSA. Blots were probed with either $\alpha$ rpl11 (Abcam, Cat. \# ab79352) or $\alpha H A$ (Sigma, H9658) antibodies. Blots were exposed on film (Amersham Hyperfilm ECL, 28906836) using Amersham ECL Western Blotting Reagent (GE, RPN2106).

\section{Fluorescence Activated Cell sorting}

For Fluorescence Activated Cell Sorting (FACS), 24 hpf AngioTag embryos were anaesthetized with Tricaine and dechorionated and deyolked. Embryos were then washed in PBS and incubated in $0.25 \%$ trypsin at room temperature with trituration until dissociated into a single cell suspension. Following embryo dissociation, trypsin was quenched with Leibovitz L-15 phenol free media with 10\% FBS (Gibco). Cells were passed through a $70 \mathrm{uM}$ strainer and collected by centrifugation at $600 \mathrm{xg}$ for $1 \mathrm{~min}$. Cells were resuspended in phenol free L-15 $+10 \% \mathrm{FBS}$, and GFP + cells were collected by FACS sorting on a BD FACSAria ${ }^{\mathrm{TM}}$ III machine using BD FACSDiva ${ }^{\mathrm{TM}}$ software (Becton Dickinson Biosciences). Sorted cells were collected at 500xg for $5 \mathrm{~min}$ and RNA was isolated and DNAse treated using the ZR-Duet kit (Zymo Research D7003).

\section{Whole-mount RNA in situ hybridization}

DIG-labeled antisense riboprobes for the below genes were generated using DIG Labeling Kit (Roche). In situ hybridization was performed as described (Swift et al., 2014). BM purple (Sigma) was used for DIG-labeled probes. Riboprobes were generated for the following genes: ENSDARG00000069998 (F' GGTGCAGATAACTGGGAAGGTGATAG， R' TCAGTGTGAAGACGTACACC), ENSDARG00000076721 (F' CAGATGAAGTAAAGTCAGTATCTGTGATG， R' GTAGTCTGGTTGGTGAATGAATAAGC), ENSDARG00000098293 (F' GACCATGTGCTGAGAAATGTGAAGAG, R' TGTTAGCTCCATTTCCGCAG), ENSDARG00000098129 (F’ GCTGTCTGTGGAGCGCTAAGTGTTTGTCT, R' TAATACGACTCACTATAGGGAGAATGTCACATCCGACCAATCAGAAT), 
ENSDARG00000093124/scpp8 (F' GATGAATACTTTGAAGGGATTGATTCTGAT, R' CATCATAGCATCAGAAATCATCAAG), ENSDARG00000008414/exoc312a (F' GAGGCTGAAGGTAGATTTGGACAGATCGAC, R' TGTGGATCCCACTATTTTTACAGTG), ENSDARG00000056643/slc22a7b.1 (F', ACAACTTTATCGCCGCCATC, R' AGCCCTCCAGTCATTCACAA), ENSDARG00000099980/bpifcl (F' CAGAAGCAGATGAAGTTCATTAGTTCATTA, CTCCATGTTAGTGACTGCTTGTTGG)

\section{Generation of constructs and transgenic lines}

The RiboTag and AngioTag constructs were generated using Gateway Technology (Kwan et al., 2007). Rpl10a was PCR amplified from $24 \mathrm{hpf}$ zebrafish cDNA using forward primer GTG AGA GGG GAG ATA TCA CG and reverse primer CTA AGC GTA ATC TCC AAC ATC GTA TGG GTA GTA GAG GCG CTG TGG TTT TCC CAT G, and TOPO TA cloned into the PCR-II vector. Bridging PCR was then used to add EGFP and viral 2A sequence (Provost et al., 2007) to generate the "RiboTag" construct pME-EGFP-2aRpl10a-3xHA. Gateway LR reactions were used to combine pDEST-IsceI-Flk7kb, pMEeGFP-2a-Rpl10a-3xHA, and p3E-polyA. The kdrl:egfp-2a-rpl10a3xHA "AngioTag" construct was digested with IsceI enzyme and microinjected into the blastomere of onecell stage zebrafish embryos. A stable $T g(k d r l: e g f p-2 a-r p l 10 a 3 x H A)^{y 530}$ germline transgenic line was established by screening through multiple generations.

The UAS:RiboTag construct was generated using SLiCE technology (Zhang et al., 2012). The pT1ump-14xUAS-MCS-POUT (Yokogawa et al., 2012) was digested with EcoRI and XhoI and then forward primer TCC CAT CGC GTC TCA GCC TCA CTT TGA GCT CCT CCA CAC GAA TTC GCC ACC ATG GTG TCA AAA G and reverse primer ACA TGT TCA GTT AAC GGT GGC TGA GAC TTA ATT ACT AGT CTC GAG TTA AGC GTA ATC TGG AAC ATC were used to slice clone the RiboTag cassette downstream from the 14x UAS sequence, using pME-eGFP-2a-Rp110a-3xHA as the template.

The Gene C (ENSDARG000098293) and Gene B (ENSDARG00000076721) mutants were generated using CRISPR-Cas9 technology, as outlined in Gagnon JA, et al (Gagnon et al., 2014). For ENSDARG00000098293, the guide primer sequence was TAATACGACTCACTATAGGAATTGGGCGACTTACTGCGTTTTAGAGCTAGAA ATAGCAAG, for ENSDARG00000076721, the guide primer sequence was TAATACGACTCACTATAGGTTTGGACCTCATGAGAGTGTTTTAGAGCTAGAA. Genotype was determined using an ABI 3130 (Applied Biosystems) with screening primers TGTAAAACGACGGCCAGTATGGCTGTAGATGAATGAAGACT and GTGTCTTTCTCAGCACATGGTCAGAGG for ENSDARG00000098293 and screening primers TGTAAAACGACGGCCAGTCAGGTGTGTTTGGTGCTGAT and GTGTCTTCACGGGCATTAACTCACCAT for ENSDARG00000076721.

The Gene $\mathrm{C}$ mutant described in this manuscript has a 20bp deletion in exon 2.

${ }^{37}$ TTGGGCGACTTAA----------------GTGAAGGAGTTAAGCGAAGCTCAGACC
${ }^{37}$ TTGGGCGACTTACTGCAGGAGTTTAATGATGTTGTGAAGGAGTTAAGCGAAGCTCAGAC

The Gene B mutant described in this manuscript has a 5bp deletion in exon 2.

${ }^{192}$ TTATCAGATACTGTGGATGTTTGGACCTCATGAG-----GATAGCTGAAATCTATAAGCA ${ }^{247}$

192TTATCAGATACTGTGGATGTTTGGACCTCATGAGAGTCGGATAGCTGAAATCTATAAGCA ${ }^{252}$ 


\section{Microscopic Imaging}

Embryos used for imaging were anesthetized using $1 \mathrm{x}$ tricaine and mounted in $0.8-1.5 \%$ low melting point agarose dissolved in embryo buffer and mounted on a depression slide. Confocal fluorescence imaging was performed with an LSM 880 (Zeiss) or a Nikon TI2 with CSUW1 spinning disk microscope.

\section{Caudal Plexus Measurements}

The dorsal-ventral width of the caudal plexus was measured under the first ISV posterior to the anal pore, using Fiji (Schindelin et al., 2012). Measurements to the left, underneath, and to the right of the ISV were taken, and then averaged and normalized to the overall dorsal-ventral height of the embryo at the first ISV posterior to the anal pore, which was measured in a similar manner to the caudal plexus. The values for each genotype were then averaged, normalized to the wild type measurement, and the standard error of the mean was determined. A student's t-test was run to determine significance.

\section{Study Approval}

Zebrafish husbandry and research protocols were reviewed and approved by the NICHD Animal Care and Use Committee at the National Institutes of Health. All animal studies were carried out according to NIH-approved protocols, in compliance with the Guide for the Care and use of Laboratory Animals.

\section{Author Contributions}

MFM, KM, NMA, and VNP performed experiments; MFM, DEG, NMA, and BMW analyzed results and made the figures; MFM, DEG, KM, and BMW designed the research and wrote the paper.

Conflict-of-interest disclosure: The authors declare no competing financial interests.

Correspondence: Brant M. Weinstein, Division of Developmental Biology, National Institute of Child Health and Human Development, National Institutes of Health, 6 Center Drive, Bethesda, MD 20892; e-mail: flyingfish2@nih.gov.

\section{Acknowledgements}

The authors would like to thank members of the Weinstein laboratory for their critical comments on this manuscript, Harold Burgess and Jennifer Sinclair for the HuC:Gal4 and Dusp27:Gal4 fish, and Tianwei Li at the NICHD MGC sequencing core for performing the library construction and RNA sequencing. This work was supported by the Intramural Research Program of the Eunice Kennedy Shriver National Institute of Child Health and Human Development, National Institutes of Health (ZIA-HD008808 and ZIA-HD001011, to BMW) and, in part, by the Intramural Research Program of the National Human Genome Research Institute, National Institutes of Health (ZIC-HG200345-10, to ADB). 


\section{FIGURE LEGENDS}

Figure 1. Affinity-tagged ribosomal protein subunits (Ribotags) can be used to purify translated RNAs in vivo

(A) Schematic diagram of the egfp-2a-rpl10a3xHA RiboTag cassette. (B) Schematic diagram of a 3xHA-tagged ribosome translating an mRNA. (C) Schematic diagram illustrating the workflow for TRAP purification of RNAs from RiboTag mRNA-injected zebrafish. (D) Western blot of starting lysates and TRAP supernatants and eluates from either control or RiboTag-injected animals, probed with $\alpha$ HA antibody. (E) Western blots of fractions collected from sucrose density gradient sedimentation of lysates from control or RiboTag-injected animals, probed with either $\alpha$ Rpl11 or $\alpha$ HA. HA-tagged ribosomes sediment together with Rpl11-positive polysomes in the RiboTag-injected samples. (F) Western blots of fractions collected from sucrose gradient sedimentation of EDTA-treated lysates from control or RiboTag-injected animals, probed with either $\alpha$ Rpl11 or $\alpha H A$. Rpl11-positive endogenous ribosomes and HA-tagged ribosomes both sediment in the 60S fraction after EDTA treatment. (G) Schematic diagram of the IsceI(kdrl:egfp-2arpl10a3xHA) transgene. (H) Composite confocal micrograph of a $3 \mathrm{dpf} T g(\mathrm{kdrl}: \text { egfp })^{l a 116}$ transgenic animal, for comparison purposes. (I) Composite confocal micrograph of a 3 dpf Tg(kdrl:egfp-2a-rpl10a3xHA) ${ }^{y 530}$ (“AngioTag”) transgenic animal. (J) Schematic diagram illustrating the workflow for TRAP purification of RNAs from AngioTag zebrafish. (K) Western blot of starting lysates and TRAP supernatants and eluates from either control or AngioTag animals, probed with $\alpha \mathrm{HA}$ antibody, showing longer (top) and shorter (bottom) exposures of the same blot. IgG LC = IgG light chain present in the pulldowns. (L) Quantitative RT-PCR measurement of the relative expression of $c d h 5, k d r l$, desmin, and ngnl in cDNA samples prepared from TRAP purified RNA from either RiboTag control (black columns) or AngioTag (white columns) animals, showing enrichment of vascular specific genes and depletion of non-vascular specific genes in the AngioTag TRAP samples.

Figure 2. Comparative workflow for preparation of endothelial-specific RNAs using fluorescence activated cell sorting (FACS) versus translating ribosome affinity purification (TRAP)

(A) Workflow for FACS of EGFP-positive endothelial cells and RNA preparation from 24 hpf $T g(k d r l: e g f p-2 a-r p l 10 a 3 x H A)^{y 530}$ AngioTag transgenic animals (sample \#4 in panel C). RNA is collected from the cells after more than $1 \frac{1}{2}$ hours of embryonic dissociation and cell sorting. (B) Workflow for TRAP of translated mRNAs from 24 hpf egfp-2arpl10a3xHA RiboTag mRNA-injected animals (sample \#2 in panel C) or Tg(kdrl:egfp-2arpl10a3xHA) ${ }^{y 530}$ (AngioTag) transgenic animals (sample \#6 in panel C). Lysate are prepared from intact animals and the RNA is stabilized immediately at the beginning of the procedure. (C) Samples collected in triplicate for RNAseq analysis. $24 \mathrm{hpf}$ RiboTag mRNA injected (samples 1 and 2), dissociated AngioTag transgenic (samples 3, 4), or AngioTag transgenic animals (samples, 5, 6). Part of each sample was used for whole lysate total RNA collection (samples 1,3, and 5). The remainder of each sample was used for either TRAP purification of total (sample 2) or endothelial (sample 6) polysome mRNA, or for FACS sorting of EGFP-positive endothelial cells followed by RNA isolation 
(sample 4). (D) Principal component analysis of RNAseq data obtained from the six sample types noted in panel $\mathrm{C}$, each run in triplicate (total of 18 samples).

\section{Figure 3. Comparing the whole-animal transcriptome and translatome}

(A) Samples collected for RNAseq analysis of whole animal translatome (sample 2) vs. whole animal transcriptome (sample 1) from $24 \mathrm{hpf}$ RiboTag mRNA injected animals. (B) Top $10 \mathrm{GO}$ terms for the highest-translated genes. (C) Percentage fold-change in the percentage usage of each codon between the most highly translated genes (fold $\geq 1.5, \mathrm{BH}$ adjusted $\mathrm{p} \leq 0.05$ comparing samples 2 vs. 1 ) and the least highly translated genes (fold $\leq$ $1.5, \mathrm{BH}$-adjusted $\mathrm{p} \leq 0.05$ comparing samples 2 vs. 1 ). The color-coding scheme shows the percentage change in the relative usage of each codon between the two sets of genes (with the usage of each codon calculated as a percentage of the total number of codons coding for a particular amino acid). (D) 3' UTR length of all Ensembl genes (first column) compared to the most highly translated (second column) and least highly translated (third column) sets of genes.

\section{Figure 4. Enrichment of endothelial genes by Fluorescence Activated Cell Sorting}

(A) Samples collected from $24 \mathrm{hpf}$ AngioTag transgenic animals dissociated into a cell suspension, for RNAseq analysis of mRNA from either FACS sorted endothelial cells (sample 4) or unsorted input cells (sample 3). (B) Volcano plot of samples 4 vs. 3. (C) Top twenty GO terms for genes most highly enriched in the FACS sorted endothelial cell samples vs. whole animal unsorted cells. Six out of the top twenty GO terms represent endothelial process-related terms (in red text, and highlighted in blue).

\section{Figure 5. Enrichment of endothelial genes by Translating Ribosome Affinity Purification}

A. Samples collected for RNAseq analysis of TRAP purified endothelial cell polysome mRNA from $24 \mathrm{hpf}$ AngioTag transgenic animals (sample 6) normalized to its input total mRNA (sample 5) compared to TRAP purified total embryonic polysome mRNA from 24 hpf RiboTag mRNA injected animals (sample 2) normalized to its input total mRNA (sample 1). (B) Volcano plot of endothelial enrichment using the sample comparison shown in panel A. (C) Top twenty GO terms for genes most highly enriched in the TRAP purified endothelial polysome mRNA samples. Fourteen out of the top twenty GO terms represent endothelial process-related terms (in red text, and highlighted in blue). (D) Log2 fold enrichment of endothelial specific genes and depletion of non-endothelial specific genes in AngioTag TRAP-RNAseq sample 6 (normalized to 5) as compared to RiboTag TRAP-RNAseq sample 2 (normalized to 1). (E) Four unannotated genes found among the top 50 genes enriched in the AngioTag TRAP-RNAseq data set (sample 6). (F) Log2 fold enrichment of the four unannotated genes noted in panel E in AngioTag TRAP-RNAseq sample 6 (normalized to 5) compared to RiboTag TRAP-RNAseq sample 2 (normalized to 1). (G-N) Whole mount in situ hybridization of $24 \mathrm{hpf}$ wild type zebrafish probed for unannotated gene A (panels G,K), gene B (panels H,L), gene C (panels I,M), and gene D (panels J,N). (O) Four annotated genes found among the top 50 genes enriched in the AngioTag TRAP-RNAseq data set (sample 6). (P) Log2 fold enrichment of the four annotated genes noted in panel $\mathrm{O}$ in AngioTag TRAP-RNAseq sample 6 (normalized to 5) 
compared to RiboTag TRAP-RNAseq sample 2 (normalized to 1). (Q-T) Whole mount in situ hybridization of $24 \mathrm{hpf}$ wild type zebrafish probed for scpp8 $(\mathrm{Q})$, exoc312a $(\mathrm{R})$, slcc22a7b.1 (S), and bpifcl (T). Images shown are lateral views of the whole animal (panels $\mathrm{G}, \mathrm{H}, \mathrm{I}, \mathrm{JQ}, \mathrm{R}, \mathrm{S}$ ), higher magnification lateral views of the trunk (panels $\mathrm{K}, \mathrm{L}, \mathrm{M}, \mathrm{N}$ ), and a dorsal view of the head with staining of the heart noted (T).

Figure 6. Mutants of unannotated genes have vascular phenotypes

(A) In $76721^{y 587}$ mutants a 5 bp deletion in ENSDARG00000076721 introduces an early stop codon, resulting in a protein truncation from 316 amino acids to 78 amino acids. (BD) Confocal microscopy of 3 dpf embryos from a $76721^{1587 /+}$ heterozygous incross showing a dilated caudal plexus in heterozygous and mutant embryos as compared to wild type siblings. (E) Graph comparing the caudal plexus height of wild type, $76721^{1587 / /}$ heterozygous, and $76721^{y 587 / 15887}$ mutant siblings. Values shown are the percent change compared to wild type ( $\mathrm{N}=41$ wild types, 58 heterozygotes, 23 mutants). (F) In $98293^{y 588}$ mutants a 20 bp deletion in ENSDARG00000098293 introduces an early stop codon, resulting in a protein truncation from 104 amino acids to 27 amino acids. (G-I) Confocal microscopy of $3 \mathrm{dpf}$ wild type and $98293^{\text {y588/y588 }}$ homozygous mutant embryos reveals decreased cranial vasculature in mutant embryos compared to wild type (white arrows). ${ }^{*}$ student's t-test $\mathrm{p}$-value $\leq 0.05$.

\section{Figure 7. Profiling gene expression in different tissues and cell types using Ribotag Reporter transgenics}

(A) Schematic diagram of the Tol2(uas:egfp-2a-rp110a2xHA) RiboTag Reporter transgene. (B) Green epifluorescence photomicrograph of a $30 \mathrm{hpf} T g$ (uas:egfp-2arpl10a2xHA) $y^{531} ; \quad \operatorname{Tg}(\text { dusp27:gal4 })^{y 241}$ double transgenic animal. (C) Green epifluorescence photomicrograph of a 3 dpf $\operatorname{Tg}(\text { uas:egfp-2a-rpl10a2xHA })^{y 531}$; $T g(f l i l a: g a l 4)^{u b s} 4$ double transgenic animal. (D) Green epifluorescence photomicrograph of a 6 dpf $T g$ (uas:egfp-2a-rpl10a2xHA) ${ }^{y 531}$; $T g(h u C: g a l 4)$ double transgenic animal. (E) Schematic diagram illustrating the workflow for TRAP purification of RNAs from RiboTag Reporter zebrafish crossed to Gal4 driver lines. (F) Quantitative RT-PCR measurement of the relative expression of the endothelial-specific $k d r l$ gene in samples prepared from TRAP purified RNA from Tg(uas:egfp-2arpl10a2xHA) ${ }^{y 531} ; \operatorname{Tg}\left(\right.$ flila:gal4) ${ }^{u b s}$ double-transgenic animals compared to RNA prepared whole embryo lysates from the same animals. (G) Quantitative RT-PCR measurement of the relative expression of the neural-specific snap 25 gene in samples prepared from TRAP purified RNA from $\operatorname{Tg}$ (uas:egfp-2a-rpl10a2xHA $)^{y 531} ; \operatorname{Tg}(\mathrm{huC}$ :gal4) double-transgenic animals compared to RNA prepared whole embryo lysates from the same animals. 
Figure 1, Miller et al.

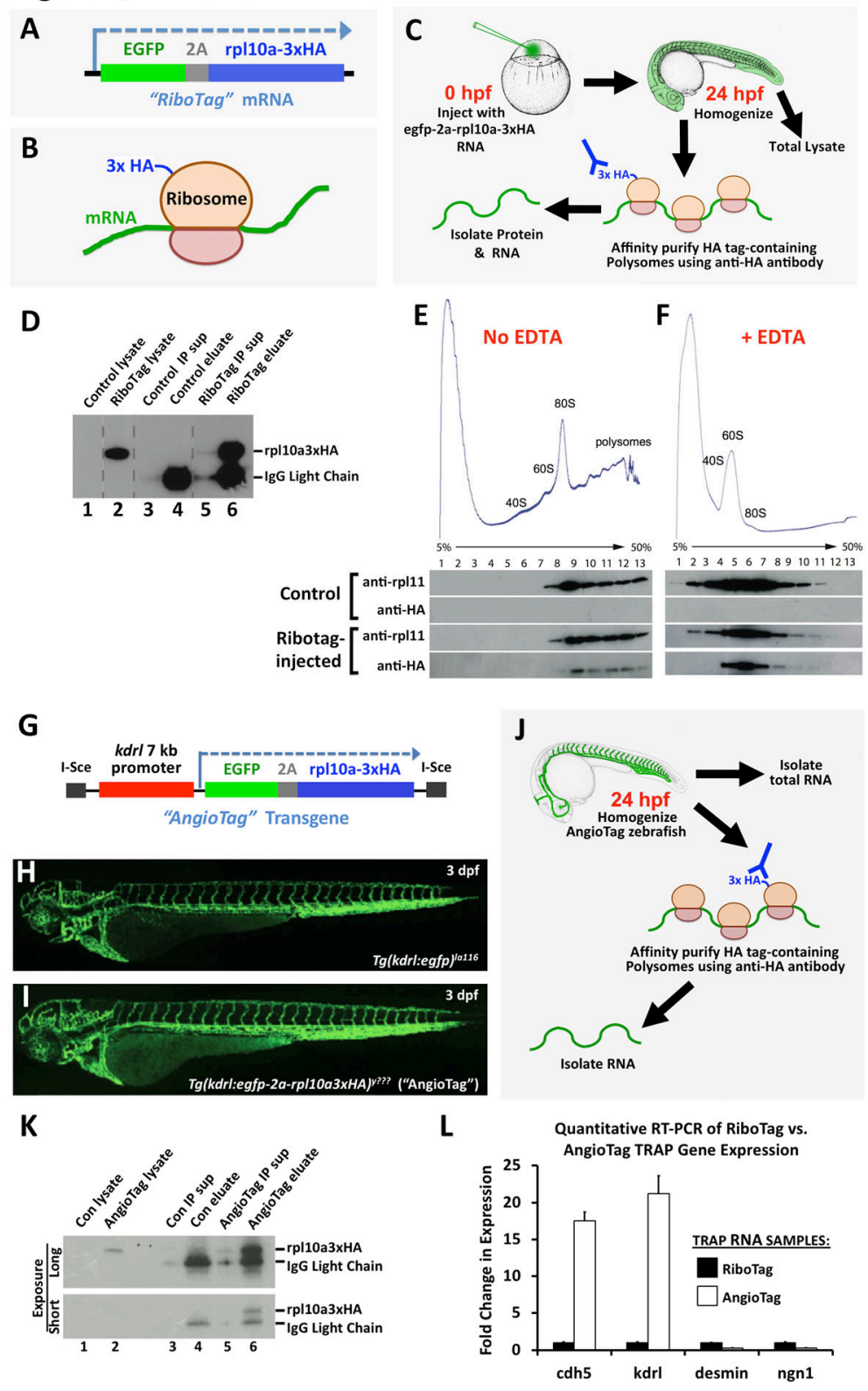




\section{Figure 2, Miller et al.}

A

\begin{tabular}{|c|}
\hline $\begin{array}{c}\text { Fluorescence Activated } \\
\text { Cell Sorting (FACS) }\end{array}$ \\
\hline
\end{tabular}

Dissociate embryos into single cells $\left(30^{\prime}\right)$ $\checkmark$

Filter, centrifuge, and resuspend cells (5-10')

$$
\checkmark
$$

FACs sort endothelial cells $\left(60^{\prime}\right)$$$
16
$$

Collect RNA
B

Translating Ribosome Affinity Purification (TRAP)

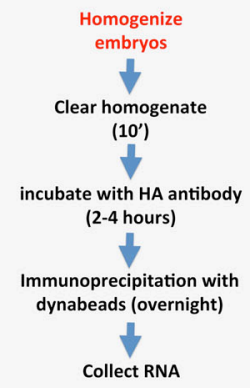

D

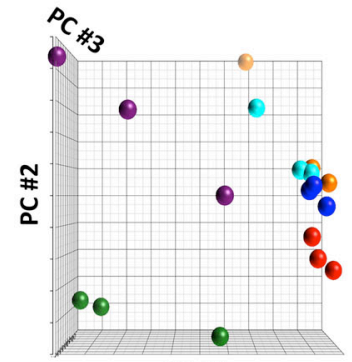

PC \#1

CRibotag Total RNA (\#1)

- facs total RNa (\#3)

FACS Sorted EC RNA (\#4)

AngioTag Total RNA (\#5)

(\#6)
C

Samples for RNAseq Analysis

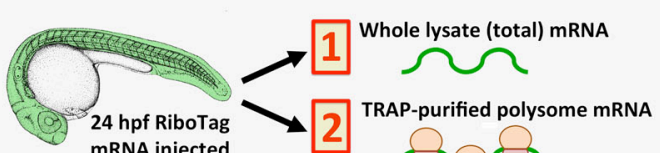

$24 \mathrm{hpf}$ RiboTag
mRNA injected

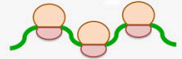

Whole lysate (total) mRNA

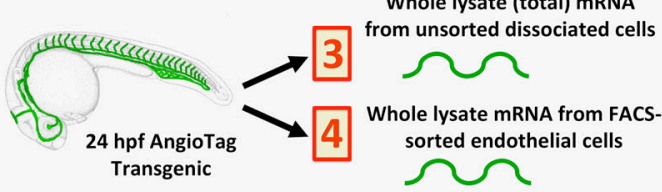

Ribotag Polysome RNA (\#2)

AngioTag EC Polysome RNA

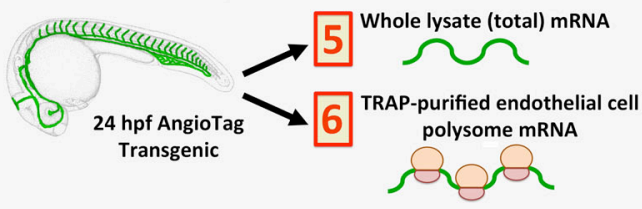




\section{Figure 3, Miller et al.}

A

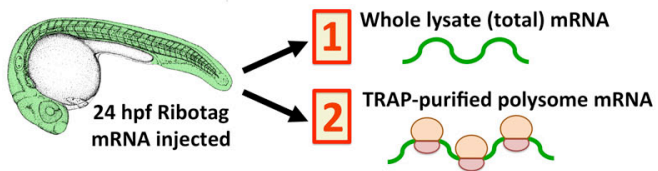

Compare 2 versus 1

B

Top 10 GO Terms for the most highly translated genes

\begin{tabular}{|l|c|c|c|}
\hline GO biological process & $\begin{array}{c}\text { \# of genes } \\
\text { in set }\end{array}$ & $\begin{array}{c}\text { Average fold } \\
\text { increase }\end{array}$ & P-value \\
\hline mRNA splice site selection (GO:0006376) & 5 & 26.18 & $5.43 E-03$ \\
\hline Spliceosomal complex assembly (GO:0000245) & 8 & 19.71 & $3.74 \mathrm{E}-05$ \\
\hline mRNA cleavage (GO:0006379) & 5 & 17.45 & $3.81 \mathrm{E}-02$ \\
\hline spliceosomal snRNP assembly (GO:0000387) & 6 & 12.57 & $3.28 \mathrm{E}-02$ \\
\hline $\begin{array}{l}\text { RNA splicing, via transesterification reactions (GO: } \\
\text { 0000375) }\end{array}$ & 27 & 10.28 & $2.99 \mathrm{E}-15$ \\
\hline mRNA splicing, via spliceosome (GO:0000398) & 26 & 9.99 & $2.58 \mathrm{E}-14$ \\
\hline $\begin{array}{l}\text { RNA splicing, via transesterification reactions with } \\
\text { bulged adenosine as nucleophile (GO:0000377) }\end{array}$ & 26 & 9.99 & $2.58 \mathrm{E}-14$ \\
\hline regulation of mRNA processing (GO:0050684) & 7 & 9.77 & $3.06 \mathrm{E}-02$ \\
\hline RNA secondary structure unwinding (GO:0010501) & 8 & 9.31 & $1.02 \mathrm{E}-02$ \\
\hline protein import into nucleus (GO:0006606) & 10 & 8.91 & $9.12 \mathrm{E}-04$ \\
\hline
\end{tabular}

C Differential Codon Usage in High vs. Low Translated Genes U C S A G Position C A G UUU UCU UaU UCU AUU ACU AaU agu Phe Ser Tyr Cys lle Thr Asn Ser A U UUC UCC UAC UGC AUC ACC AAC AGC Phe Ser Tyr Cys lle Thr Asn Ser U UUA UCA UAA UGA AUA ACA AAA AGA U Leu Ser Stop Stop lle Thr Lys Arg UUG UCG UAG UGG AUG ACG AAG AGG $\begin{array}{llllllllll}\text { Leu } & \text { Ser Stop Trp } & \text { Met } & \text { Thr } & \text { Arg }\end{array}$ ธั. CUU CCU CAU CGU C Leu Pro His Arg C CUC CCC CAC CGC Leu pro his arg C CUA CCA CAA CGA Leu Pro Gln Arg C CUG CCG CAG CGG

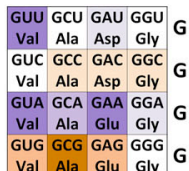

High/Low Usage Dfferential

$-50 \%$ to $-30 \%$ $-29 \%$ to $-15 \%$ $-15 \%$ to $-5 \%$ $-5 \%$ to $5 \%$ $5 \%$ to $15 \%$ $15 \%$ to $30 \%$ $30 \%$ to $50 \%$

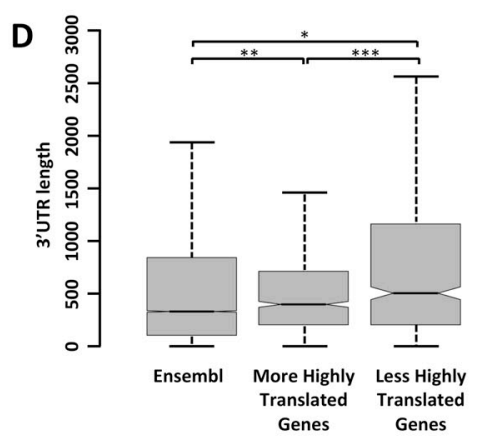




\section{Figure 4, Miller et al.}

A

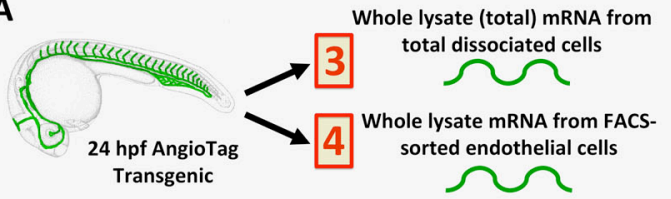

Compare 4 versus 3

C

Top 20 GO Terms for the most highly FACS-enriched genes

\begin{tabular}{|c|c|c|c|}
\hline GO biological process & $\begin{array}{c}\text { \# of genes in } \\
\text { set }\end{array}$ & $\begin{array}{l}\text { Average fold } \\
\text { increase }\end{array}$ & P-value \\
\hline vesicle docking involved in exocytosis (GO:0006904) & 11 & 5.49 & $2.56 \mathrm{E}-02$ \\
\hline endothelial cell migration (GO:0043542) & 12 & 5.44 & $1.10 \mathrm{E}-02$ \\
\hline regulation of vesicle fusion (GO:0031338) & 18 & 4.72 & 3.64E-04 \\
\hline activation of GTPase activity (GO:0090630) & 17 & 4.46 & $1.75 \mathrm{E}-03$ \\
\hline endosomal transport (GO:0016197) & 28 & 3.99 & 5.40E-06 \\
\hline vacuolar transport (GO:0007034) & 35 & 3.88 & $1.15 \mathrm{E}-07$ \\
\hline peptidyl-tyrosine autophosphorylation (GO:0038083) & 15 & 3.84 & $4.61 \mathrm{E}-02$ \\
\hline lymph vessel development (GO:0001945) & 18 & 3.66 & $1.27 \mathrm{E}-02$ \\
\hline vacuole organization (GO:0007033) & 27 & 3.54 & $1.14 \mathrm{E}-04$ \\
\hline embryonic hemopoiesis (GO:0035162) & 21 & 3.43 & $5.73 \mathrm{E}-03$ \\
\hline regulation of small GTPase mediated signal transduction (GO:0051056) & 43 & 3.25 & $1.88 \mathrm{E}-07$ \\
\hline regulation of Ras protein signal transduction (GO:0046578) & 38 & 3.16 & $5.00 \mathrm{E}-06$ \\
\hline myeloid cell homeostasis (GO:0002262) & 23 & 3.1 & $1.02 \mathrm{E}-02$ \\
\hline blood vessel morphogenesis (G0:0048514) & 82 & 3.07 & $1.21 \mathrm{E}-14$ \\
\hline blood vessel development (GO:0001568) & 94 & 3.05 & $6.72 \mathrm{E}-17$ \\
\hline erythrocyte homeostasis (GO:0034101) & 22 & 3.05 & 2.13E-02 \\
\hline regulation of Rho protein signal transduction (GO:0035023) & 25 & 2.97 & 8.27E-03 \\
\hline positive regulation of GTPase activity (GO:0043547) & 25 & 2.97 & 8.27E-03 \\
\hline vasculature development (GO:0001944) & 106 & 2.94 & 3.44E-18 \\
\hline angiogenesis (GO:0001525) & 60 & 2.92 & $2.92 \mathrm{E}-09$ \\
\hline
\end{tabular}

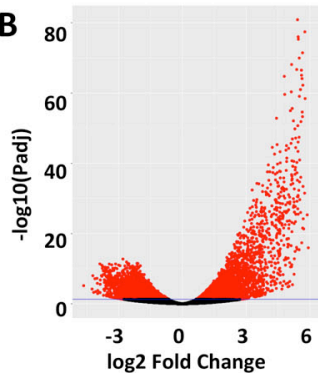

log2 Fold Change 
Figure 5, Miller et al.

A

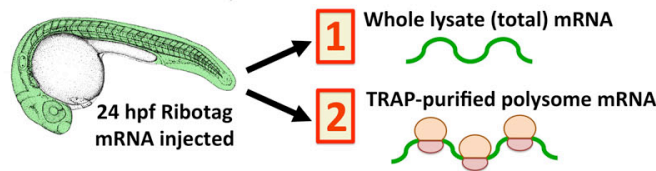

Compare 2 normalized to 1 (total translatome) versus 6 normalized to 5 (EC translatome)

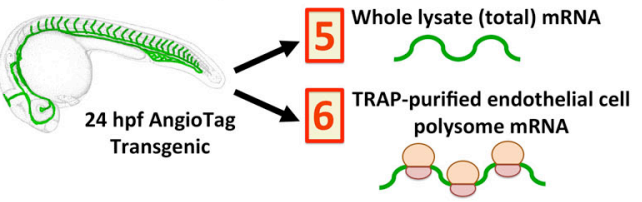

B

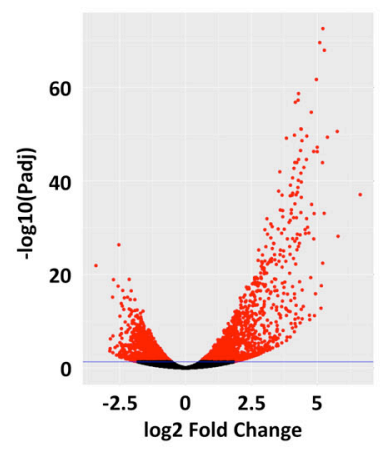

E Unannotated Genes

Gene Ensembl ID Symbol

A ENSDARG00000069998 si:ch211-145b13.6

B ENSDARG00000076721 zgc:171686

C ENSDARG00000098293 si:dkey-27i16.2

D ENSDARG00000098129 si:dkeyp-97a10.2

$\mathbf{F}$

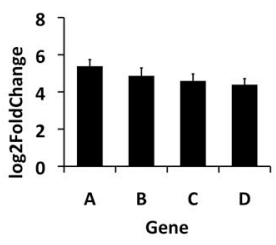

0

Annotated Genes

\begin{tabular}{cc} 
Ensembl ID & Symbol \\
\hline ENSDARG00000093124 & scpp8 \\
\hline ENSDARG00000008414 & exoc312a \\
\hline ENSDARG00000056643 & slc22a7b.1 \\
\hline ENSDARG00000099980 & bpifcl \\
\hline
\end{tabular}

$\mathbf{P}$

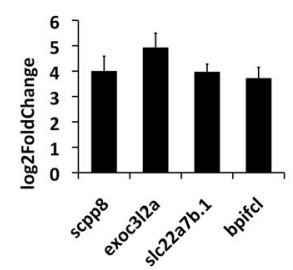

C Top 20 GO Terms for the most highly enriched genes

\begin{tabular}{|l|c|c|c|}
\hline GO biological process complete & \# of genes & Average fold & \\
regulation of neutrophil migration (GO:1902622) & P-value \\
\hline blood vessel endothelial cell migration (GO:0043534) & 6 & 11.79 & $4.74 \mathrm{E}-02$ \\
\hline endothelial cell migration (GO:0043542) & 8 & 8.38 & $2.22 \mathrm{E}-02$ \\
\hline negative regulation of immune system process (GO:0002683) & 10 & 7.14 & $6.73 \mathrm{E}-03$ \\
\hline vasculogenesis (GO:0001570) & 10 & 6.55 & $1.43 \mathrm{E}-02$ \\
\hline lymphangiogenesis (GO:0001946) & 12 & 5.61 & $4.70 \mathrm{E}-04$ \\
\hline lymph vessel morphogenesis (GO:0036303) & 12 & 5.55 & $8.98 \mathrm{E}-03$ \\
\hline lymph vessel development (GO:0001945) & 16 & 5.13 & $8.98 \mathrm{E}-03$ \\
\hline sprouting angiogenesis (GO:0002040) & 21 & 4.78 & $2.66 \mathrm{E}-04$ \\
\hline endothelium development (GO:0003158) & 12 & 4.71 & $4.47 \mathrm{E}-02$ \\
\hline blood vessel morphogenesis (GO:0048514) & 70 & 4.14 & $6.55 \mathrm{E}-19$ \\
\hline embryonic hemopoiesis (GO:0035162) & 16 & 4.12 & $9.90 \mathrm{E}-03$ \\
\hline angiogenesis (GO:0001525) & 52 & 3.99 & $7.04 \mathrm{E}-13$ \\
\hline blood vessel development (GO:0001568) & 77 & 3.94 & $8.66 \mathrm{E}-20$ \\
\hline vasculature development (GO:0001944) & 88 & 3.85 & $2.85 \mathrm{E}-22$ \\
\hline cardiovascular system development (GO:0072358) & 88 & 3.77 & $1.22 \mathrm{E}-21$ \\
\hline circulatory system development (GO:0072359) & 124 & 2.82 & $1.84 \mathrm{E}-20$ \\
\hline regulation of cell motility (GO:2000145) & 27 & 2.81 & $8.87 \mathrm{E}-03$ \\
\hline endocytosis (GO:0006897) & 29 & 2.8 & $4.28 \mathrm{E}-03$ \\
\hline regulation of cell migration (GO:0030334) & 25 & 2.77 & $2.62 \mathrm{E}-02$ \\
\hline
\end{tabular}

D

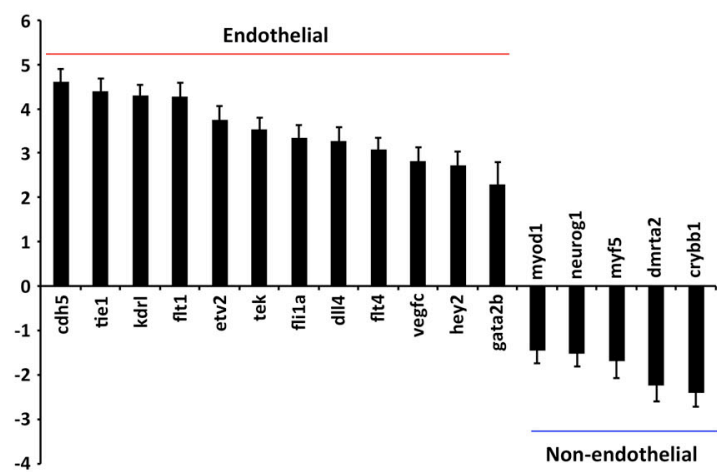

G

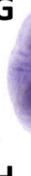

H

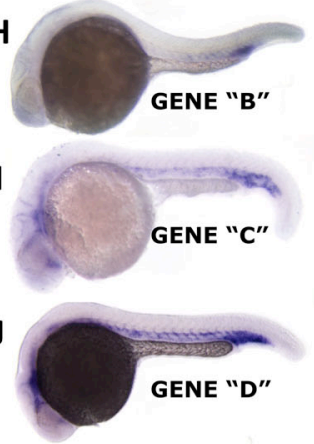

Q

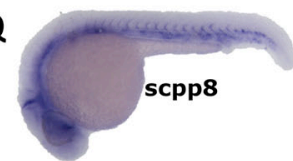

$\mathbf{R}$

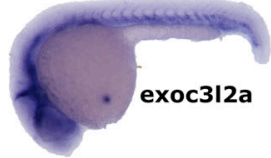

K

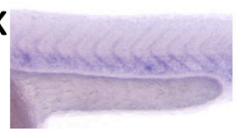

$\mathbf{L}$

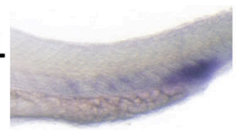

M

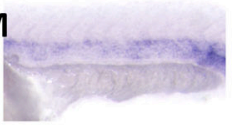

N
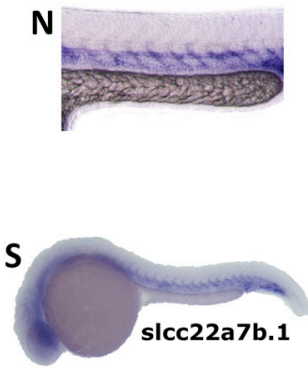

T

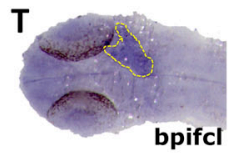




\section{Figure 6, Miller et al.}

GENE B

(ENSDARG00000076721)

A 71 ...FGPHESRIAE...

...FGPHEDS*

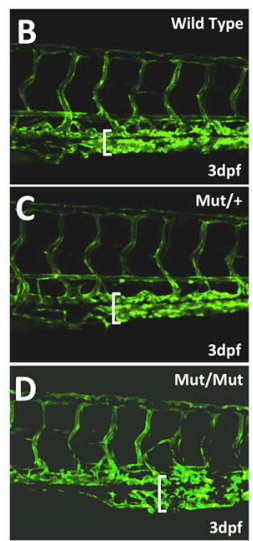

E Caudal Venous Plexus (CVP) Widt

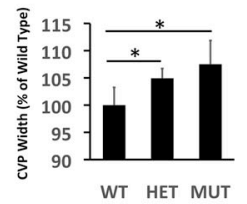

GENE C

(ENSDARG00000098293)

F $\quad$...DLLQEFNDVVKE... ...DLSEGVKRSSDL*
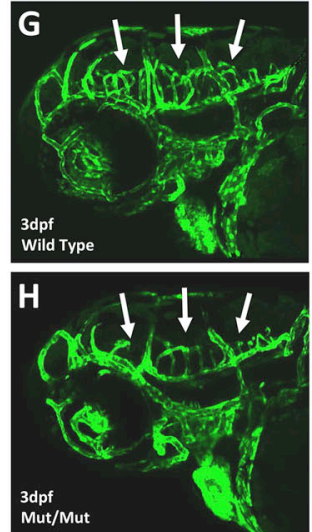

I

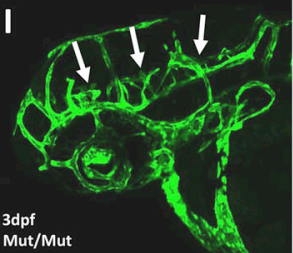


Figure 7, Miller et al.

A
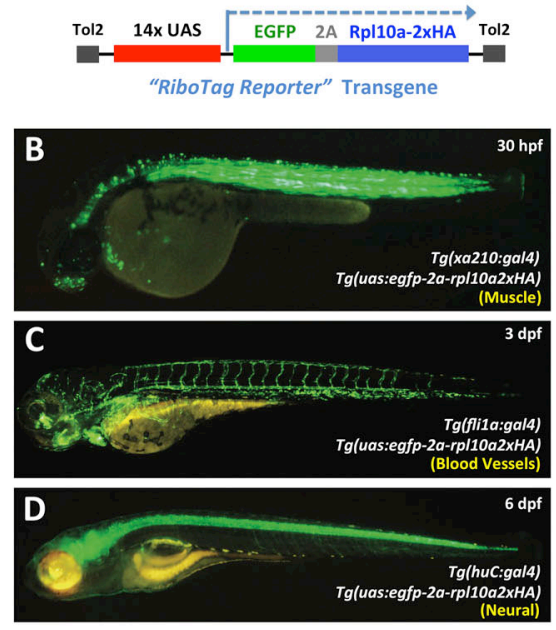

E

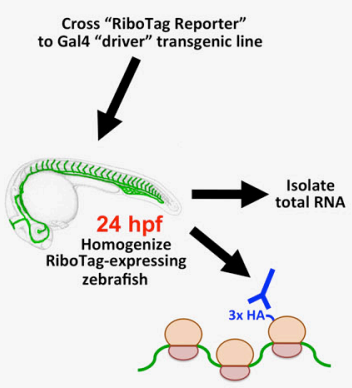

Affinity purify HA tag-containing -HA antibody

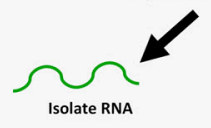

F

Quantitative RT-PCR of $k d r l$ in Fli:Gal4, UAS:Ribo2 $x$ double transgenic animals

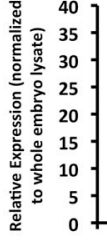

G Quantitative RT-PCR of snap25 in HuC:Gal4,

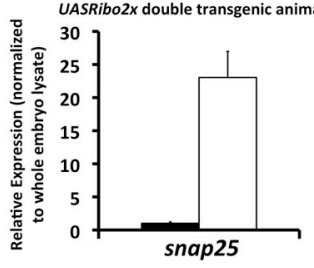


Supplemental Table 1

\begin{tabular}{|l|l|l|l|l|l|}
\hline Sample & $\begin{array}{c}\text { Total No.\# } \\
\text { of reads }\end{array}$ & $\begin{array}{c}\text { No.\# reads } \\
\text { mapping } \\
\text { uniquely }\end{array}$ & $\begin{array}{c}\% \text { reads } \\
\text { mapping } \\
\text { uniquely }\end{array}$ & $\begin{array}{c}\text { No.\# reads } \\
\text { mapped to } \\
\text { multiple } \\
\text { loci }\end{array}$ & $\begin{array}{c}\text { \% reads } \\
\text { mapped to } \\
\text { multiple } \\
\text { loci }\end{array}$ \\
\hline 1-1 RiboTag Whole Lysate & 49956134 & 42588557 & $85.25 \%$ & 2969994 & $5.95 \%$ \\
\hline 1-2 RiboTag Whole Lysate & 69239038 & 59221672 & $85.53 \%$ & 3076200 & $4.44 \%$ \\
\hline 1-3 RiboTag Whole Lysate & 47632455 & 41583642 & $87.30 \%$ & 2029387 & $4.26 \%$ \\
\hline 2-1 RiboTag TRAP & 50661654 & 34986862 & $69.06 \%$ & 3703631 & $7.31 \%$ \\
\hline 2-2 RiboTag TRAP & 56252261 & 43023522 & $76.48 \%$ & 3810600 & $6.77 \%$ \\
\hline 2-3 RiboTag TRAP & 45403187 & 36331265 & $80.02 \%$ & 2344794 & $5.16 \%$ \\
\hline 3-1 AngioTag FACS Total Cells & 55085749 & 51341850 & $93.20 \%$ & 1651529 & $3.00 \%$ \\
\hline 3-2 AngioTag FACS Total Cells & 53707170 & 49094355 & $91.41 \%$ & 1495389 & $2.78 \%$ \\
\hline 3-3 AngioTag FACS Total Cells & 58235304 & 51189179 & $87.90 \%$ & 2147287 & $3.69 \%$ \\
\hline 4-1 AngioTag FACS ECs & 56759660 & 50662241 & $89.26 \%$ & 1443422 & $2.54 \%$ \\
\hline 4-2 AngioTag FACS ECs & 55032277 & 31439590 & $57.13 \%$ & 20211551 & $36.73 \%$ \\
\hline 4-3 AngioTag FACS ECs & 52123159 & 45630183 & $87.54 \%$ & 2572154 & $4.93 \%$ \\
\hline 5-1 AngioTag Whole Lysate & 46722553 & 40175213 & $85.99 \%$ & 1951949 & $4.18 \%$ \\
\hline 5-2 AngioTag Whole Lysate & 57354200 & 53786329 & $93.78 \%$ & 1898238 & $3.31 \%$ \\
\hline 5-3 AngioTag Whole Lysate & 53618024 & 50073486 & $93.39 \%$ & 1900801 & $3.55 \%$ \\
\hline 6-1 AngioTag TRAP & 49463459 & 26997399 & $54.58 \%$ & 7640140 & $15.45 \%$ \\
\hline 6-2 AngioTag TRAP & 58760419 & 54794280 & $93.25 \%$ & 2027329 & $3.45 \%$ \\
\hline 6-3 AngioTag TRAP & 55289491 & 51098279 & $92.42 \%$ & 2198844 & $3.98 \%$ \\
\hline
\end{tabular}


Supplemental Table 2

\begin{tabular}{|l|c|c|c|c|}
\hline & & & & \\
GO biological process & Fold enrichement FACs & Fold enrichment AngioTag & p-value FACS & p-value AngioTag \\
\hline endothelial cell migration (GO:0043542) & 5.44 & 7.14 & 2.92 & $6.73 \mathrm{E}-03$ \\
\hline lymph vessel development (GO:0001945) & 3.66 & 5.13 & $1.27 \mathrm{E}-02$ & $6.01 \mathrm{E}-04$ \\
\hline blood vessel morphogenesis (GO:0048514) & 3.07 & 4.14 & $1.21 \mathrm{E}-14$ & $6.55 \mathrm{E}-19$ \\
\hline blood vessel development (GO:0001568) & 3.05 & 3.94 & $6.72 \mathrm{E}-17$ & $8.66 \mathrm{E}-20$ \\
\hline vasculature development (GO:0001944) & 2.94 & 3.85 & $3.44 \mathrm{E}-18$ & $2.85 \mathrm{E}-22$ \\
\hline angiogenesis (GO:0001525) & 2.92 & 3.99 & & $2.92 \mathrm{E}-09$ \\
\hline
\end{tabular}




\section{REFERENCES}

Allison, M.B., Patterson, C.M., Krashes, M.J., Lowell, B.B., Myers, M.G., Jr., and Olson, D.P. (2015). TRAP-seq defines markers for novel populations of hypothalamic and brainstem LepRb neurons. Mol Metab 4, 299-309.

Brichta, L., Shin, W., Jackson-Lewis, V., Blesa, J., Yap, E.L., Walker, Z., Zhang, J., Roussarie, J.P., Alvarez, M.J., Califano, A., et al. (2015). Identification of neurodegenerative factors using translatome-regulatory network analysis. Nat Neurosci 18 , 1325-1333.

Cannon, J.E., Place, E.S., Eve, A.M., Bradshaw, C.R., Sesay, A., Morrell, N.W., and Smith, J.C. (2013). Global analysis of the haematopoietic and endothelial transcriptome during zebrafish development. Mech Dev 130, 122-131.

Carmeliet, P., Ferreira, V., Breier, G., Pollefeyt, S., Kieckens, L., Gertsenstein, M., Fahrig, M., Vandenhoeck, A., Harpal, K., Eberhardt, C., et al. (1996). Abnormal blood vessel development and lethality in embryos lacking a single VEGF allele. Nature 380, 435-439.

Childs, S., Chen, J.N., Garrity, D.M., and Fishman, M.C. (2002). Patterning of angiogenesis in the zebrafish embryo. Development 129, 973-982.

Choi, J., Dong, L., Ahn, J., Dao, D., Hammerschmidt, M., and Chen, J.N. (2007). FoxH1 negatively modulates flk1 gene expression and vascular formation in zebrafish. Dev Biol 304, 735-744.

Covassin, L., Amigo, J.D., Suzuki, K., Teplyuk, V., Straubhaar, J., and Lawson, N.D. (2006). Global analysis of hematopoietic and vascular endothelial gene expression by tissue specific microarray profiling in zebrafish. Dev Biol 299, 551-562.

Davison, J.M., Akitake, C.M., Goll, M.G., Rhee, J.M., Gosse, N., Baier, H., Halpern, M.E., Leach, S.D., and Parsons, M.J. (2007). Transactivation from Gal4-VP16 transgenic insertions for tissue-specific cell labeling and ablation in zebrafish. Dev Biol 304, 811-824.

Dumont, D.J., Fong, G.H., Puri, M.C., Gradwohl, G., Alitalo, K., and Breitman, M.L. (1995). Vascularization of the mouse embryo: a study of flk-1, tek, tie, and vascular endothelial growth factor expression during development. Dev Dyn 203, 80-92.

Fang, Y., Gupta, V., Karra, R., Holdway, J.E., Kikuchi, K., and Poss, K.D. (2013). Translational profiling of cardiomyocytes identifies an early Jak1/Stat3 injury response required for zebrafish heart regeneration. Proc Natl Acad Sci U S A 110, 13416-13421.

Fero, K., Bergeron, S.A., Horstick, E.J., Codore, H., Li, G.H., Ono, F., Dowling, J.J., and Burgess, H.A. (2014). Impaired embryonic motility in dusp27 mutants reveals a developmental defect in myofibril structure. Dis Model Mech 7, 289-298.

Ferrara, N., Carver-Moore, K., Chen, H., Dowd, M., Lu, L., O'Shea, K.S., Powell-Braxton, L., Hillan, K.J., and Moore, M.W. (1996). Heterozygous embryonic lethality induced by targeted inactivation of the VEGF gene. Nature 380, 439-442.

Fong, G.H., Rossant, J., Gertsenstein, M., and Breitman, M.L. (1995). Role of the Flt-1 receptor tyrosine kinase in regulating the assembly of vascular endothelium. Nature 376, 66-70. 
Gagnon, J.A., Valen, E., Thyme, S.B., Huang, P., Akhmetova, L., Pauli, A., Montague, T.G., Zimmerman, S., Richter, C., and Schier, A.F. (2014). Efficient mutagenesis by Cas9 protein-mediated oligonucleotide insertion and large-scale assessment of single-guide RNAs. PLoS One 9, e98186.

Gonzalez, C., Sims, J.S., Hornstein, N., Mela, A., Garcia, F., Lei, L., Gass, D.A., Amendolara, B., Bruce, J.N., Canoll, P., et al. (2014). Ribosome profiling reveals a celltype-specific translational landscape in brain tumors. J Neurosci 34, 10924-10936.

Gore, A.V., Monzo, K., Cha, Y.R., Pan, W., and Weinstein, B.M. (2012). Vascular development in the zebrafish. Cold Spring Harb Perspect Med 2, a006684.

Horstick, E.J., Jordan, D.C., Bergeron, S.A., Tabor, K.M., Serpe, M., Feldman, B., and Burgess, H.A. (2015). Increased functional protein expression using nucleotide sequence features enriched in highly expressed genes in zebrafish. Nucleic Acids Res 43, e48.

Housley, M.P., Reischauer, S., Dieu, M., Raes, M., Stainier, D.Y., and Vanhollebeke, B. (2014). Translational profiling through biotinylation of tagged ribosomes in zebrafish. Development 141, 3988-3993.

Huber, T.L., Kouskoff, V., Fehling, H.J., Palis, J., and Keller, G. (2004). Haemangioblast commitment is initiated in the primitive streak of the mouse embryo. Nature 432, 625-630.

Hupe, M., Li, M.X., Gertow Gillner, K., Adams, R.H., and Stenman, J.M. (2014). Evaluation of TRAP-sequencing technology with a versatile conditional mouse model. Nucleic Acids Res 42, e14.

Jin, S.W., Beis, D., Mitchell, T., Chen, J.N., and Stainier, D.Y. (2005). Cellular and molecular analyses of vascular tube and lumen formation in zebrafish. Development 132, 5199-5209.

Jung, H.M., Isogai, S., Kamei, M., Castranova, D., Gore, A.V., and Weinstein, B.M. (2016). Imaging blood vessels and lymphatic vessels in the zebrafish. Methods Cell Biol 133, 69-103.

Kamei, M., Saunders, W.B., Bayless, K.J., Dye, L., Davis, G.E., and Weinstein, B.M. (2006). Endothelial tubes assemble from intracellular vacuoles in vivo. Nature 442, 453456.

Kawakami, K., Abe, G., Asada, T., Asakawa, K., Fukuda, R., Ito, A., Lal, P., Mouri, N., Muto, A., Suster, M.L., et al. (2010). zTrap: zebrafish gene trap and enhancer trap database. BMC Dev Biol 10, 105.

Kimura, Y., Satou, C., and Higashijima, S. (2008). V2a and V2b neurons are generated by the final divisions of pair-producing progenitors in the zebrafish spinal cord. Development 135, 3001-3005.

Kwan, K.M., Fujimoto, E., Grabher, C., Mangum, B.D., Hardy, M.E., Campbell, D.S., Parant, J.M., Yost, H.J., Kanki, J.P., and Chien, C.B. (2007). The Tol2kit: a multisite gateway-based construction kit for Tol2 transposon transgenesis constructs. Dev Dyn 236, 3088-3099. 
Lai, K., Amsterdam, A., Farrington, S., Bronson, R.T., Hopkins, N., and Lees, J.A. (2009). Many ribosomal protein mutations are associated with growth impairment and tumor predisposition in zebrafish. Dev Dyn 238, 76-85.

Lawson, N.D., Scheer, N., Pham, V.N., Kim, C.H., Chitnis, A.B., Campos-Ortega, J.A., and Weinstein, B.M. (2001). Notch signaling is required for arterial-venous differentiation during embryonic vascular development. Development 128, 3675-3683.

Leslie, J.D., Ariza-McNaughton, L., Bermange, A.L., McAdow, R., Johnson, S.L., and Lewis, J. (2007). Endothelial signalling by the Notch ligand Delta-like 4 restricts angiogenesis. Development 134, 839-844.

Mardinly, A.R., Spiegel, I., Patrizi, A., Centofante, E., Bazinet, J.E., Tzeng, C.P., MandelBrehm, C., Harmin, D.A., Adesnik, H., Fagiolini, M., et al. (2016). Sensory experience regulates cortical inhibition by inducing IGF1 in VIP neurons. Nature 531, 371-375.

Marquart, G.D., Tabor, K.M., Brown, M., Strykowski, J.L., Varshney, G.K., LaFave, M.C., Mueller, T., Burgess, S.M., Higashijima, S., and Burgess, H.A. (2015). A 3D Searchable Database of Transgenic Zebrafish Gal4 and Cre Lines for Functional Neuroanatomy Studies. Front Neural Circuits 9, 78.

Mi, H., Muruganujan, A., Casagrande, J.T., and Thomas, P.D. (2013). Large-scale gene function analysis with the PANTHER classification system. Nat Protoc 8, 1551-1566.

Pham, V.N., Roman, B.L., and Weinstein, B.M. (2001). Isolation and expression analysis of three zebrafish angiopoietin genes. Dev Dyn 221, 470-474.

Provost, E., Rhee, J., and Leach, S.D. (2007). Viral 2A peptides allow expression of multiple proteins from a single ORF in transgenic zebrafish embryos. Genesis 45, 625-629.

Sanz, E., Evanoff, R., Quintana, A., Evans, E., Miller, J.A., Ko, C., Amieux, P.S., Griswold, M.D., and McKnight, G.S. (2013). RiboTag analysis of actively translated mRNAs in Sertoli and Leydig cells in vivo. PLoS One 8, e66179.

Sanz, E., Yang, L., Su, T., Morris, D.R., McKnight, G.S., and Amieux, P.S. (2009). Celltype-specific isolation of ribosome-associated mRNA from complex tissues. Proc Natl Acad Sci U S A 106, 13939-13944.

Sato, T.N., Tozawa, Y., Deutsch, U., Wolburg-Buchholz, K., Fujiwara, Y., GendronMaguire, M., Gridley, T., Wolburg, H., Risau, W., and Qin, Y. (1995). Distinct roles of the receptor tyrosine kinases Tie-1 and Tie-2 in blood vessel formation. Nature 376, 70-74.

Schindelin, J., Arganda-Carreras, I., Frise, E., Kaynig, V., Longair, M., Pietzsch, T., Preibisch, S., Rueden, C., Saalfeld, S., Schmid, B., et al. (2012). Fiji: an open-source platform for biological-image analysis. Nat Methods 9, 676-682.

Schwanhausser, B., Busse, D., Li, N., Dittmar, G., Schuchhardt, J., Wolf, J., Chen, W., and Selbach, M. (2011). Global quantification of mammalian gene expression control. Nature 473, 337-342.

Scott, E.K., Mason, L., Arrenberg, A.B., Ziv, L., Gosse, N.J., Xiao, T., Chi, N.C., Asakawa, K., Kawakami, K., and Baier, H. (2007). Targeting neural circuitry in zebrafish using GAL4 enhancer trapping. Nat Methods 4, 323-326. 
Shalaby, F., Rossant, J., Yamaguchi, T.P., Gertsenstein, M., Wu, X.F., Breitman, M.L., and Schuh, A.C. (1995). Failure of blood-island formation and vasculogenesis in Flk-1deficient mice. Nature 376, 62-66.

Shigeoka, T., Jung, H., Jung, J., Turner-Bridger, B., Ohk, J., Lin, J.Q., Amieux, P.S., and Holt, C.E. (2016). Dynamic Axonal Translation in Developing and Mature Visual Circuits. Cell 166, 181-192.

Soden, M.E., Miller, S.M., Burgeno, L.M., Phillips, P.E., Hnasko, T.S., and Zweifel, L.S. (2016). Genetic Isolation of Hypothalamic Neurons that Regulate Context-Specific Male Social Behavior. Cell Rep 16, 304-313.

Sumanas, S., Jorniak, T., and Lin, S. (2005). Identification of novel vascular endothelialspecific genes by the microarray analysis of the zebrafish cloche mutants. Blood 106, 534541.

Swift, M.R., Pham, V.N., Castranova, D., Bell, K., Poole, R.J., and Weinstein, B.M. (2014). SoxF factors and Notch regulate nr2f2 gene expression during venous differentiation in zebrafish. Dev Biol 390, 116-125.

Swift, M.R., and Weinstein, B.M. (2009). Arterial-venous specification during development. Circ Res 104, 576-588.

Torres-Vazquez, J., Gitler, A.D., Fraser, S.D., Berk, J.D., Van, N.P., Fishman, M.C., Childs, S., Epstein, J.A., and Weinstein, B.M. (2004). Semaphorin-plexin signaling guides patterning of the developing vasculature. Dev Cell 7, 117-123.

Tryon, R.C., Pisat, N., Johnson, S.L., and Dougherty, J.D. (2013). Development of translating ribosome affinity purification for zebrafish. Genesis 51, 187-192.

Vogel, C., Abreu Rde, S., Ko, D., Le, S.Y., Shapiro, B.A., Burns, S.C., Sandhu, D., Boutz, D.R., Marcotte, E.M., and Penalva, L.O. (2010). Sequence signatures and mRNA concentration can explain two-thirds of protein abundance variation in a human cell line. Mol Syst Biol 6, 400.

Vogeli, K.M., Jin, S.W., Martin, G.R., and Stainier, D.Y. (2006). A common progenitor for haematopoietic and endothelial lineages in the zebrafish gastrula. Nature 443, 337-339.

Westerfield, M. (2000). The Zebrafish Book: A Guide for the Laboratory Use of Zebrafish (Danio rerio). 4th edn Eugene: University of Oregon Press.

Wiegers, U., and Hilz, H. (1972). Rapid isolation of undegraded polysomal RNA without phenol. FEBS Lett 23, 77-82.

Yokogawa, T., Hannan, M.C., and Burgess, H.A. (2012). The dorsal raphe modulates sensory responsiveness during arousal in zebrafish. J Neurosci 32, 15205-15215.

Yu, J.A., Castranova, D., Pham, V.N., and Weinstein, B.M. (2015). Single-cell analysis of endothelial morphogenesis in vivo. Development 142, 2951-2961.

Zhang, Y., Werling, U., and Edelmann, W. (2012). SLiCE: a novel bacterial cell extractbased DNA cloning method. Nucleic Acids Res 40, e55.

Zygmunt, T., Gay, C.M., Blondelle, J., Singh, M.K., Flaherty, K.M., Means, P.C., Herwig, L., Krudewig, A., Belting, H.G., Affolter, M., et al. (2011). Semaphorin-PlexinD1 
bioRxiv preprint doi: https://doi.org/10.1101/815696; this version posted October 27, 2019. The copyright holder for this preprint (which was not certified by peer review) is the author/funder. All rights reserved. No reuse allowed without permission.

AngioTag expression profiling

Miller et al.

signaling limits angiogenic potential via the VEGF decoy receptor sFlt1. Dev Cell 21, 301314. 